\title{
MAPK pathway mutations in head and neck cancer affect immune microenvironments and ErbB3 signaling
}

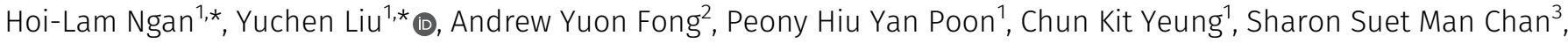

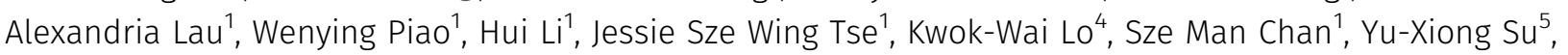

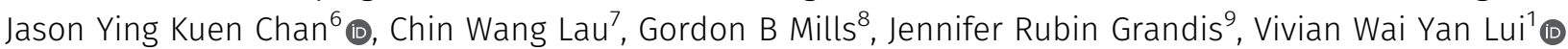

\begin{abstract}
MAPK pathway mutations affect one-fifth of head and neck squamous cell carcinoma (HNSCC). Unexpectedly, MAPK pathway aberrations are associated with remarkably long patient survival, even among patients with TP53 mutations (median $\sim 14 \mathrm{yr}$ ). We explored underlying outcome-favoring mechanisms with omics followed by preclinical models. Strikingly, multiple hotspot and non-hotspot MAPK mutations (A/BRAF, HRAS, MAPK1, and $M A P 2 K 1 / 2)$ all abrogated ErbB3 activation, a well-established HNSCC progression signal. Inhibitor studies functionally defined ERK activity negatively regulating phospho-ErbB3 in MAPKmutants. Furthermore, pan-pathway immunoprofiling investigations identified MAPK-mutant tumors as the only " $\mathrm{CDB}^{+} \mathrm{T}$-cellinflamed" tumors inherently bearing high-immunoreactive, constitutive cytolytic tumor microenvironments. Immunocompetent MAPK-mutant HNSCC models displayed active cell death and

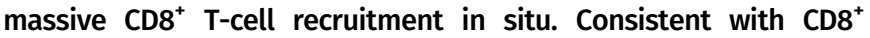
T-inflamed phenotypes, MAPK-mutant HNSCC patients, independent of tumor-mutational burden, survived 3.3-4 times longer than WT patients with anti-PD1/PD-L1 immunotherapies. Similar prognosticity was noted in pan-cancers. We uncovered clinical, signaling, and immunological uniqueness of MAPK-mutant HNSCC with potential biomarker utilities predicting favorable patient survival.
\end{abstract}

DOI 10.26508/Isa.201900545 | Received 9 September 2019 | Revised 17 April 2020 | Accepted 17 April 2020 | Published online 7 May 2020

\section{Introduction}

The incidence of head and neck squamous cell carcinoma (HNSCC) is approaching 0.7 million/year globally (Vigneswaran \& Williams, 2014). Despite extensive genomic and molecular characterizations of HNSCC primary tumors, the clinical significance of somatic aberrations in HNSCC remains underexplored. As of today, we still lack clinically relevant genomic biomarkers for effective management of HNSCC patients. This is largely due to our lack of understanding of the precise tumor biology and potential clinical utility of the genomic signatures of patients' tumors.

For HNSCC, recent genomic studies have revealed that human papillomavirus (HPV) positivity in patients' tumors can be potentially used as a favorable prognostic biomarker (mainly for cancer from the oropharynx subsite), which can clinically stratify patients for treatment de-intensification purposes (Gillison et al, 2019; Mehanna et al, 2019; Pearlstein et al, 2019). The biological reasons underlying HPV's positive prognosticity have been unfolded and found to be linked to the antigenic nature of HPV and the tumor's TP53 wild-type (WT) status which allows proper execution of druginduced apoptosis by functional p53 (Fakhry \& Gillison, 2006). Similarly, genomic studies showed that somatic TP53 mutations could robustly predict poor HNSCC patient outcome, for reasons that mutant p53 could cause drug resistance and radiation resistance due to biological impairment of cancer cell apoptosis in HNSCC. However, the high frequency of TP53 mutations in $>80-85 \%$ of primary HNSCC greatly limits their development into useful stratification biomarkers for treatment selection, especially because TP53-mutant HNSCC cannot be effectively drugged. Recently, PIK3CA mutations have been shown to be predictive of PI3K inhibitor and nonsteroidal anti-inflammatory drug (NSAID) responses in HNSCC, with proven biology demonstrated in PI3K-mutant, PI3Kactivated preclinical models of HNSCC and retrospective patient cohorts (Lui et al, 2013; Hedberg et al, 2019). These studies identified drug sensitivity characteristics of PI3K-addicted tumors in HNSCC. Yet, clinical incorporation of PIK3CA mutations as candidatepredictive biomarkers for clinical utility still awaits further

\footnotetext{
${ }^{1}$ School of Biomedical Sciences, Faculty of Medicine, The Chinese University of Hong Kong, Hong Kong, Hong Kong SAR ${ }^{2}$ Department of Biochemistry, Case Western Reserve University, Cleveland, OH, USA ${ }^{3}$ Faculty of Medicine, Imperial College London, London, UK ${ }^{4}$ Department of Anatomical and Cellular Pathology, Faculty of Medicine, The Chinese University of Hong Kong, Hong Kong, Hong Kong SAR ${ }^{5}$ Department of Oral and Maxillofacial Surgery, Faculty of Dentistry, The University of Hong Kong, Hong Kong, Hong Kong SAR ${ }^{6}$ Department of Otorhinolaryngology, Head and Neck Surgery, Faculty of Medicine, The Chinese University of Hong Kong, Hong Kong, Hong Kong SAR ${ }^{7}$ Department of Otorhinolaryngology Head and Neck, Yan Chai Hospital, Hong Kong, Hong Kong SAR ${ }^{8}$ Department of Cell, Development and Cancer Biology, Knight Cancer Institute, Oregon Health and Sciences University, Portland, OR, USA ${ }^{9}$ Department of Otolaryngology-Head and Neck Surgery, University of California, San Francisco, San Francisco, CA, USA
} 
prospective validation in clinical trials. These recent findings demonstrate that a deeper understanding of the clinical impacts of HNSCC genetic aberrations in relation to their underlying biology can potentially reveal new approaches for clinical management of HNSCC.

Here, we first reported that MAPK pathway mutations in HNSCC predict remarkably long patient survival, even among patients bearing TP53 mutations (median 14 yr), much longer than that of HPV-positive HNSCC (median $\sim 5.5 \mathrm{yr}$ ). The favorable prognosticity of MAPK pathway mutations in HNSCC was found to be independent of HPV. Subsequent molecular dissections revealed two plausible underlying mechanisms operative by MAPK mutations in patient tumors, followed by preclinical HNSCC models. First, multiple hotspot and non-hotspot MAPK mutations (A/BRAF, HRAS, MAPK1, and MAP2K1/2) all abrogated ErbB3 activation, an established signal for HNSCC progression. Inhibitor studies functionally defined ERK activity negatively regulating $p$-ErbB3 in MAPK-mutants. Second, comprehensive pan-pathway immune landscape investigations identified MAPK-mutant tumors as the only "CD8 ${ }^{+} \mathrm{T}$-cell-inflamed" tumors with inherently immunoreactive tumor microenvironments with constitutive cytolysis, subsequently validated in immunocompetent HNSCC models. As low tumoral phospho-ErbB3 levels and elevated $\mathrm{CD}^{+}$ T-cell infiltrations are recently established events driving good patient survivals in HNSCC (Takikita et al, 2011; de Ruiter et al, 2017), our study first defined somatic MAPK pathway mutations as novel genomic events governing both important outcome-favoring features in HNSCC. Consistent with the identified CD8 ${ }^{+} \mathrm{T}$-cell-inflamed nature, MAPK pathway mutations alone, independent of tumor mutational burden (TMB), were able to identify subsets of HNSCC patients benefiting significantly from anti-PD1/PD-L1 immunotherapy clinically. Similar prognosticity of MAPK pathway mutations were noted in pan-cancer immunotherapy settings. Our study uncovers novel clinical, biological, and immunological uniqueness of MAPK-mutant HNSCC and may indicate important clinical utilities of MAPK pathway mutations in this cancer.

\section{Results}

MAPK (ERK) pathway-mutated HNSCC patients have remarkably long survivals in two independent cohorts

As of today, the clinical significance of somatic genomic aberrations in HNSCC remains underexplored. Here, using a comprehensive panpathway approach, we examined the prognostic impact of mutational events of seven major cancer-related signaling pathways on HNSCC patient survivals with The Cancer Genome Atlas (TCGA) dataset ( $N=508$, exome and clinical data, June 2019). These include three major mitogenic pathways, the PI3K, MAPK(ERK), and JAK/STAT pathways; two differentiation-related pathways, the Notch and TGF- $\beta /$ Smad pathways; the inflammation-related NF-KB pathway; and the stem cellrelated WNT pathway, representing a total of $81 \%$ of cases (Fig $1 A$ and $B$ ). Pathway members are defined in the Materials and Methods section. About 17\% (84/502) of patients are HPV-positive, whereas the remaining 19\% lack these pathway aberrations or are HPV-negative/unknown.

Among all seven pathways examined, only mutations in the MAPK pathway (denoted in red for favorable survival) and the Notch pathway (blue for unfavorable survival) are associated with patient outcomes (Figs 1B and S1A-F). Mutational burdens of all pathwayaltered cases are similar ( $P=$ n.S.; Fig S1G). Our finding that Notch pathway-mutated HNSCC patients have worsened outcomes are consistent with a recent Taiwanese report that NOTCH1 mutations predict recurrences with poor outcomes (Liu et al, 2016). Unexpectedly, MAPK pathway mutations, comprising mainly activating hotspot mutations (e.g., HRAS p.G12S and MAPK1 p.E322K [Stransky et al, 2011; Van Allen et al, 2015]; Fig S2), are associated with a doubling of overall survival (OS) with a median of 95.27 versus 47.93 mo for MAPK-WT patients (log-rank test, $P=0.0201$; Fig 1C). These patients also have a reduced risk of death versus WT patients $(\mathrm{OR}=0.5466$, $P=0.0156$, Fisher's exact test).

Most strikingly, MAPK pathway mutations are also prognostic for markedly improved survival even among TP53-mutated patients (given TP53 mutations are usually indicators for HNSCC disease progression and disease aggressiveness) (Fig 1D). In fact, "MAPK and TP53 double mutant" patients $(\mathrm{N}=363)$ have an extreme long median OS of 169.25 mo ( 14 yr), which is 4.77 times longer than that of the "MAPK-WT/TP53mutated" counterparts (35.45 mo; $P=0.0074)$. The double mutant patients also have a $55.26 \%$ reduction in chances of death $(O R=0.4474)$ versus MAPK-WT counterparts ( $P=0.0063$, Fisher's exact test).

Clinically, MAPK pathway mutations are not associated with HPV status, nor clinical staging ( $P=$ n.s.), but potentially associated with lower alcohol intake per TCGA alcohol history, and a higher occurrence in females ( $P=0.01003,0.03372$, respectively, Table S1). Importantly, unlike HPV-positive HNSCC with favorable outcomes, MAPK pathway mutations span multiple head and neck anatomic subsites, including the oral cavity sites, larynx, oropharynx, and others (Table S2). More than $87 \%$ (83/95 cases) of MAPK pathway-mutated tumors are HPV-negative. Upon HPV stratification, MAPK pathway mutations are still found to be prognostic for HPV-negative HNSCC $(P=0.0352$, Fig S3). Interestingly, overexpressions of the MAPK proteome components are also prognostically associated with improved patient survival in HNSCC and 10 additional cancer types (Fig 1E and Table S3), suggesting that MAPK pathway activity may influence patient outcome in HNSCC, and likely in other cancers.

HNSCC patients in TCGA dataset were treated with conventional therapies. Strikingly, in an independent MSK-IMPACT cohort with heavily pretreated advanced and metastatic HNSCC, we cross-validated that MAPK pathway mutations (with nine MAPK pathway members sequenced therein: $H / N / K-R A S, A / B-R A F, R A F-1, M A P 2 K 1 / 2$, and MAPK1) were also prognostic of favorable HNSCC patient outcome (Fig 1F). Although only "targeted therapies" were generally described for this MSK-IMPACT study (Zehir et al, 2017), the apparent cross-cohort, cross-treatment prognostic nature of MAPK mutations seem to suggest inherent outcome-favoring features of these tumors contributive to improved patient survival.

Interestingly, MAPK pathway mutations also predict favorable outcomes in TCGA uterine corpus endometrial carcinoma (Fig 1G), indicating that the positive prognosticity of MAPK pathway aberrations is not restricted to HNSCC.

\section{Multiple MAPK hotspot and non-hotspot mutations all inhibit ErbB3 phosphorylation in HNSCC}

ErbB3 activation contributes to HNSCC proliferation (O-charoenrat et al, 2002; Zhang et al, 2014) and is a key mediator of HNSCC 
A
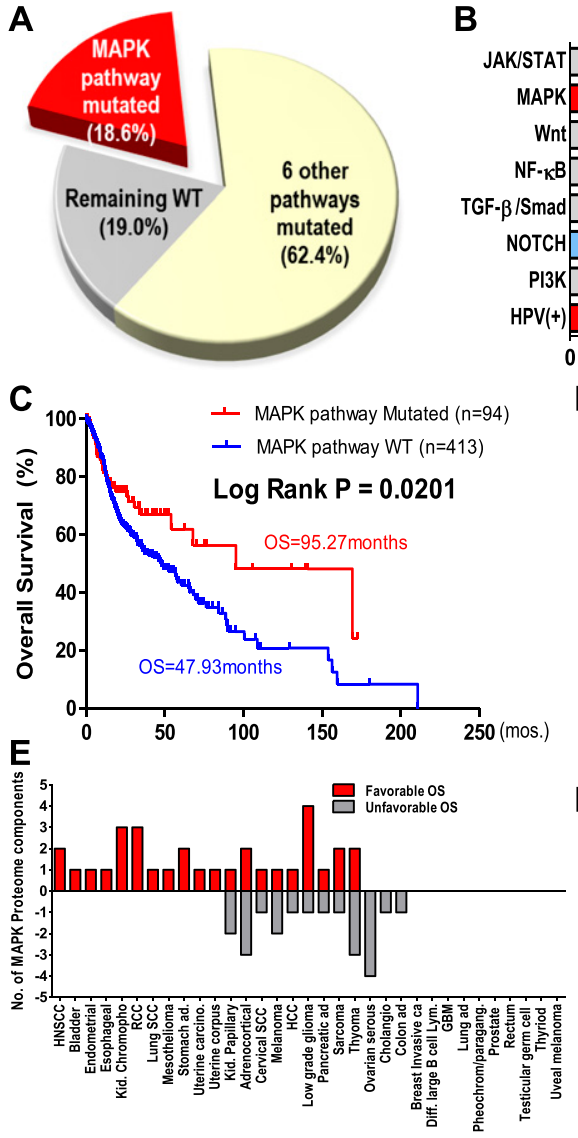

G $\quad \longrightarrow$ MAPK pathway mutated (UCEC; $n=84$ )

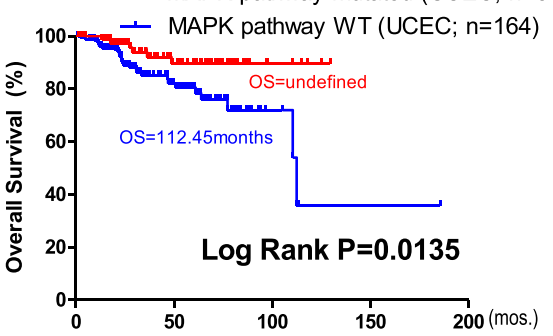

B

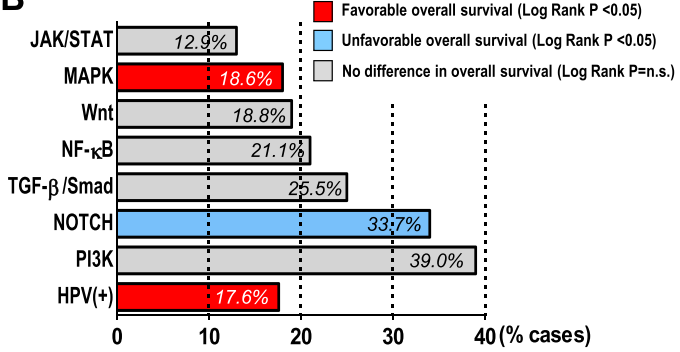

D

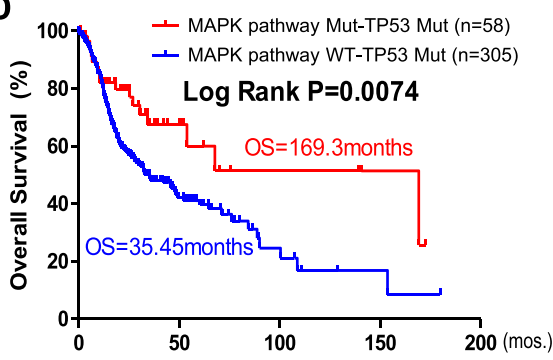

F $\longrightarrow$ MAPK pathway Mutated (MSK-IMPACT HNSCC; $\mathrm{n}=17$ ) - MAPK pathway WT (MSK-IMPACT HNSCC; $n=129$ )

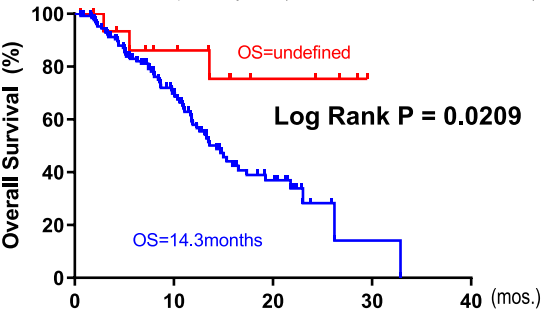

Figure 1. MAPK pathway mutations in head and neck squamous cell carcinoma (HNSCC) are associated with remarkable patient survival.

(A) Percentage of patients affected by MAPK pathway mutations and mutations of six other cancer pathways (PI3K, JAK/STAT, Notch, WNT, NF-KB, and TGF $\beta / \mathrm{Smad}$ ) in TCGA HNSCC provisional cohort ( $\mathrm{N}=$ 510). (B) HNSCC patient outcome associations for all seven pathway mutations (i.e., pathway-mutated versus pathway WT) and HPV status. Red bars indicate favorable HNSCC overall survival (OS), whereas the blue bar indicates unfavorable OS when the pathway components are mutated (log-rank test $P$-values are shown). (C) Kaplan-Meier OS curves for MAPK pathway-mutated HNSCC patients versus MAPK pathway WT patients (TCGA HNSCC provisional cohort). (D) Kaplan-Meier OS curves for TP53-mutated patients with MAPK pathway-mutated versus WT HNSCC (TCGA HNSCC provisional cohort). (E) Bar graph showing the number of MAPK pathway protein components for each cancer type (total 33 cancer types) that were significantly correlated with OS. Red bars indicate associations with favorable outcomes, whereas grey bars indicate associations with unfavorable outcomes in each cancer type, when the MAPK protein component(s) is/are overexpressed (median cutoff; The Cancer Protein Atlas database). (F) Kaplan-Meier OS curves for MAPK pathwaymutated HNSCC patients versus MAPK pathway WT patients (MSK-IMPACT HNSCC cohort). (G) Kaplan-Meier survival curves showing increased OS for uterine corpus endometrial carcinoma patients with MAPK pathway mutation versus WT (TCGA uterine corpus endometrial carcinoma cohort). progression. Data from The Cancer Protein Atlas (TCPA, https:// tcpaportal.org/tcpa/, (Li et al, 2013, 2017a)) have established tumoral overexpression of phospho-ErbB3(Y1289) as the top 1 proteinsignaling event most significantly associated with decreased HNSCC patient survival among 273 key signaling proteins examined ( $P=0.0006$, Fig $2 \mathrm{~A}$ and Table $\mathrm{S} 4$ shows top 20 signaling proteins of significant survival correlations). Overexpressions are defined as levels above median in TCPA. Based on our recent findings that MAPK1 mutation can alter ErbB family signaling in HNSCC (Van Allen et al, 2015), we thus examined the effects of various major hotspot MAPK pathway mutations on p-ErbB3(Y1289) expression. Strikingly, we found that introduction of BRAF p.V600E hotspot mutation in HNSCC cell model, FaDu, strongly inhibited p-ErbB3(Y1289) expression versus control ( $77.8 \%$ inhibition, $P=0.0038$, Fig 2B). Furthermore, many other major hotspot mutations, including HRAS p.G12V, MAPK1 p.E322K/p.D321N, MAP2K1 p.K57N, and MAP2K2 p.F57L mutations all inhibited p-ErbB3(Y1289) expressions in HNSCC cell models (Figs 2B and S4A). For ARAF, both ARAF p.P508L (non-hotspot and HNSCC associated) and the hotspot ARAF p.S214F mutations inhibited p-ErbB3(Y1289) expressions. In addition to these mutants, overexpression of either MAP2K1-WT or BRAF-WT was also sufficient to inhibit p-ErbB3(Y1289) expressions by $\sim 60 \%$ and $50 \%$, respectively.

\section{Mutant-specific ErbB3 inhibition reversed by GDC-0994}

To this end, MAPK-activating mutations function to inhibit ErbB3 activation in isogenic HNSCC models, as assessed by p-ErbB3(Y1289) levels. Therefore, we further tested this non-canonical MAPK/ErbB3 relationship using the pharmacological GDC-0994 MAPK1/2-RSK inhibitor in HNSCC cells harboring endogenous MAPK pathway mutations. As shown in Figs $2 \mathrm{C}$ and $\mathrm{S} 4 \mathrm{~B}$, only MAPK-mutant HNSCC cells showed increases in p-ErbB3(Y1289) upon inhibitor treatment 
A

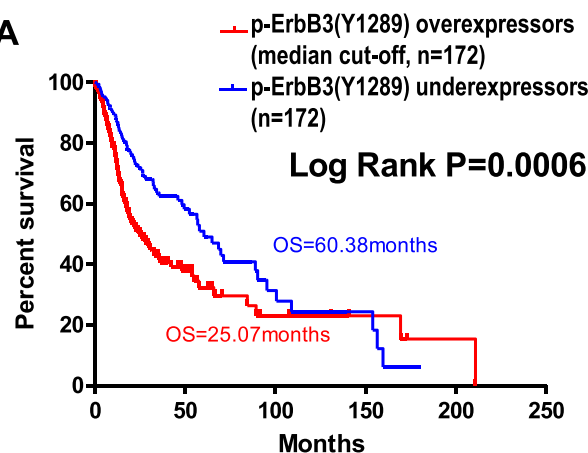

B

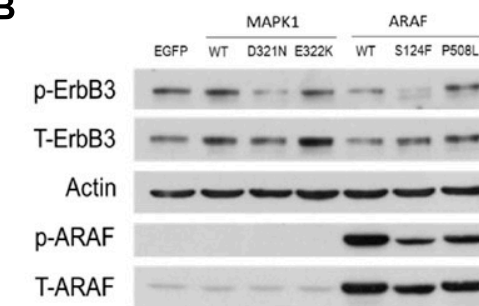

T-MAPK $1 / 3==$

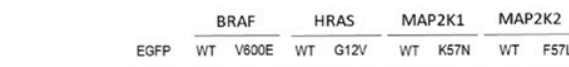

p-ErbB3 -...- - - -

T-ErbB3 $\rightarrow+\cdots \cdots$

Actin - - - - - -

p-MAP2K1 - $-\div-\cdots---$

T-MAP2K1 - - - - -

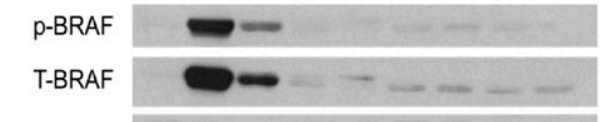

Ras

p-MAPK1/3 $==-===0$

T-MAPK $1 / 3=$

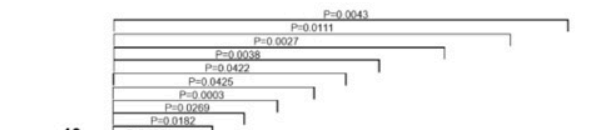

p-MAPK $1 / 3=--=$

C Pt.25 Primary cells (MAPK-mut)

GDC-0994: Veh $0.5 \mu \mathrm{M}$

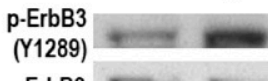

ErbB3

p-RSK

RSK

Actin
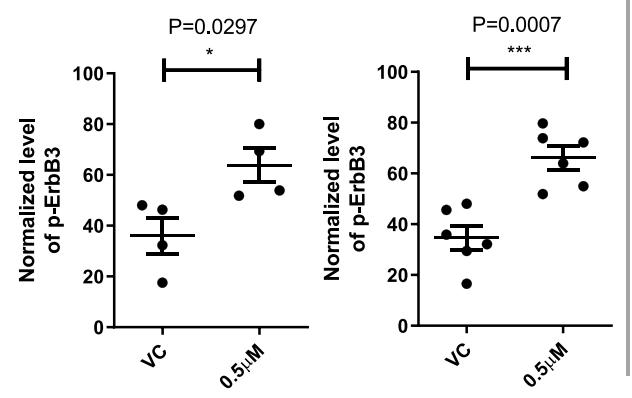

HSC-6 cell line

Veh $0.5 \mu \mathrm{M}$

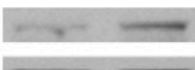

$\stackrel{-\infty}{-\infty}$

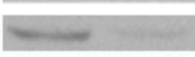

$\longrightarrow$

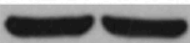

$\mathrm{P}=0.0007$

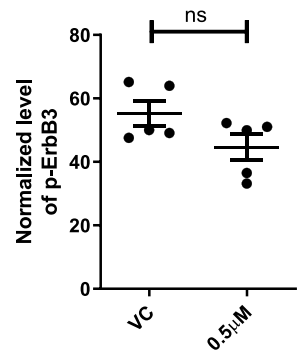

D
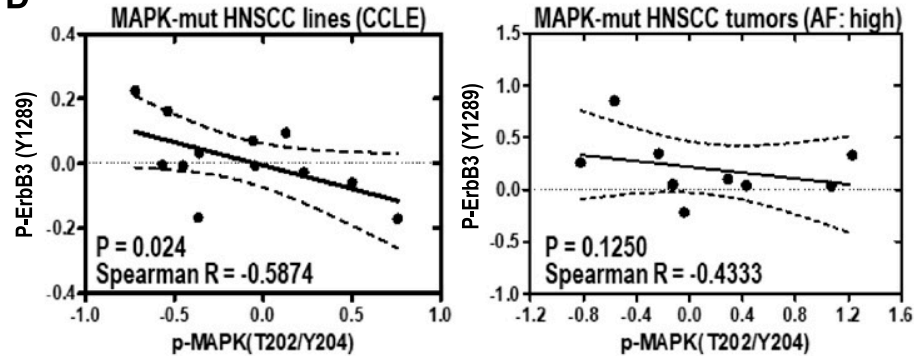

E

p-ErbB3 (Y1289) IHC

MAPK pathway mutated
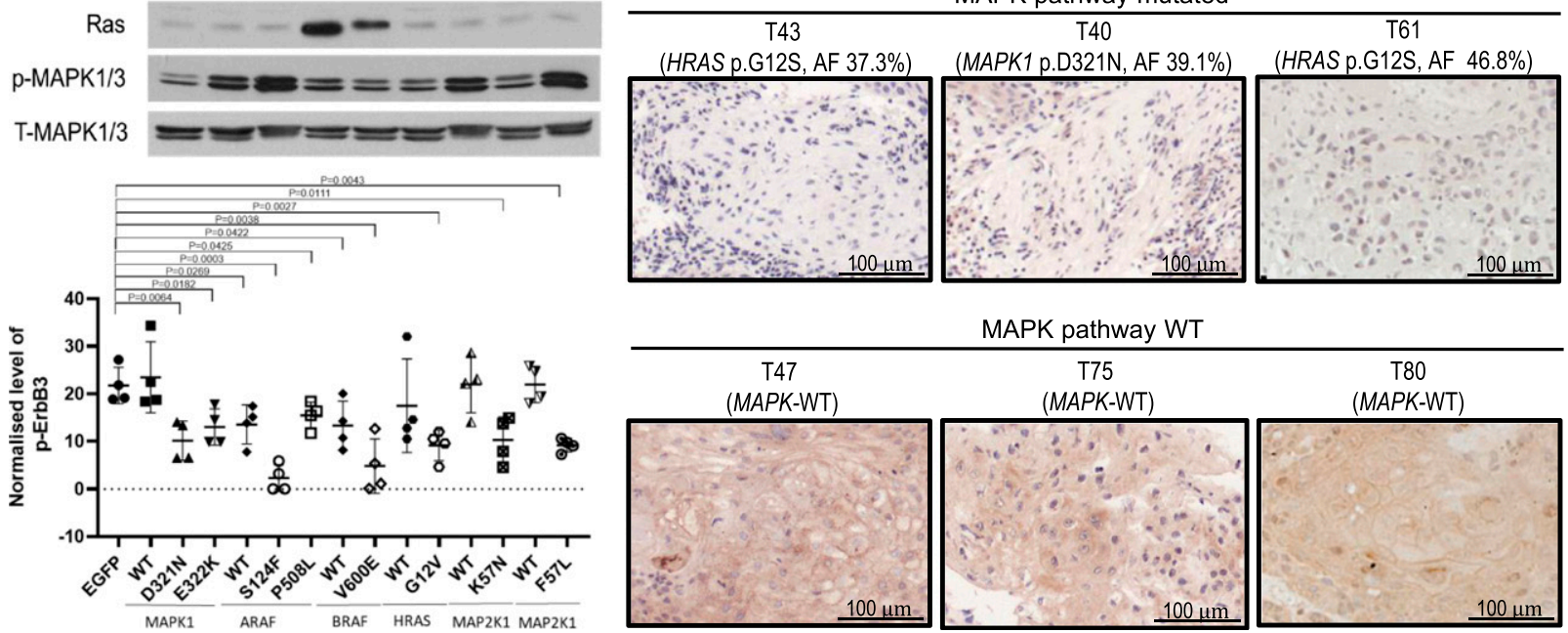

MAPK pathway WT

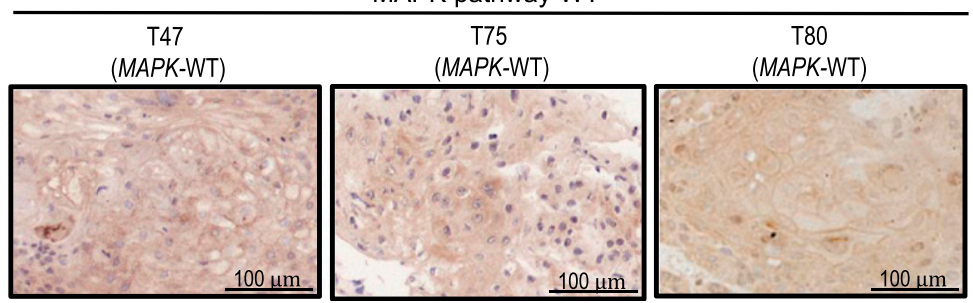

Figure 2. Multiple MAPK pathway mutations inhibited ErbB3 activation.

(A) Kaplan-Meier overall survival curves for head and neck squamous cell carcinoma (HNSCC) patients, whose tumors overexpressed versus underexpressed phosphoErbB3(Y1289) (median cutoff) in TCPA HNSCC cohort ( $N$ = 344). (B) Western blot results of phospho-ErbB3(Y1289) protein levels upon ectopic expression of the MAPK1, ARAF, BRAF, HRAS, and MAP2K1 as well as MAP2K2 wild-type and mutant constructs in FaDu cells by retroviral infection (pool of at least four independent repeats). (C) Western blot results of phospho-ErbB3(Y1289) levels of HNSCC Pt.25 primary tumor cultures (carrying both HRAS p.G12S and MAPK1 p.R135K mutations), HSC-6 cell line (carrying MAPK1 p.E322K mutation), and HSC-4 (MAPK pathway WT, Cancer Cell Line Encyclopedia [CCLE]) upon MAPK inhibitor GDC-0994 treatment for 30 min. $50 \mu g$ of protein lysate 
versus vehicle. In a primary patient culture, Pt-25 harboring MAPK1 p.R135K and HRAS p.G12S mutations and the HSC-6 cell line harboring MAPK1 p.E322K, >100-200\% increases of p-ErbB3 expression were observed, whereas such an increase was not observed in MAPK-WT cells (HSC-4). The data suggested MAPK (ERK) activity negatively regulates $\mathrm{p}$-ErbB3 in a mutant-specific manner in HNSCC. Such a mutant-specific phenomenon was further supported by proteomic findings in MAPK-mutant HNSCC cell lines ( $\mathrm{N}=12$, Cancer Cell Line Encyclopedia [CCLE] [Ghandi et al, 2019]) and in relatively homogeneous MAPK-mutant patient tumors with high allele frequencies (>40\%), in which p-MAPK(T202/Y204) and p-ErbB3 levels were negatively correlated with high Spearman $\mathrm{R}$ correlation coefficients of $-0.5874(P=0.0244)$ and $0.4333(P=0.1250$, a trend in non-microdissected TCPA tumors; Fig 2D), respectively. In MAPK-WT HNSCC tumors or cell lines, p-MAPK(T202/Y204) and p-ErbB3 were not correlated ( $P$ = n.s.; Fig S5). Thus, it is likely that when activating MAPK mutations are present at high allele frequencies in HNSCC tumors, they could substantially inhibit p-ErbB3 expressions in HNSCC. Indeed, immunohistochemistry showed three out of three MAPK-mutant HNSCC patient tumors with high allele frequencies ( 40\%) all expressed low tumorous p-ErbB3(Y1289), whereas WT tumors show high membranous p-ErbB3(Y1289) staining (Fig 2E). Therefore, MAPK pathway mutations in HNSCC function to inhibit ErbB3 signaling, an established critical signaling dictating poor HNSCC patient survival.

\section{Treatment-naive MAPK-mutant HNSCC tumors have remarkably active cytolytic immune landscapes}

Response to therapy is an important factor determining cancer patient survival. Yet, there was no clear association between MAPK mutations and responses to cisplatin, docetaxel, 5-flurouracil, methotrexate, and cetuximab in CCLE HNSCC drug-sensitivity database (Fig S6A). Similarly, expression of MAPK pathway-mutants in HNSCC cells did not significantly alter chemosensitivity (Fig S6B).

In addition to our proteomics findings, we further examine, by transcriptomic analysis, if MAPK-mutant HNSCC tumors harbor immune features favoring survival as recent findings in melanoma showed that patients with MAPK pathway mutations have remarkable clinical outcome likely due to increased neo-antigenicity or antitumor immune microenvironment (Cadley et al, 2018; Veatch et al, 2018). We compared the transcriptional profiles of MAPK pathway-mutated and WT HNSCC tumors (all treatment-naive tumors per TCGA standard). As shown in Fig 3A and Supplemental Data 1, there were 1,793 significant differentially expressed genes (DEGS) in MAPK-mutated versus WT HNSCC tumors with P-values < 0.05 and false discovery rate $(F D R)<0.05$, among which 130 proteincoding DEGs showed a $>1.41$-fold difference ( $\log _{2}$ fold-change $>0.5$ ) in gene expression with FDR < 0.05 (Fig 3B). Strikingly, more than 70\% (91/ 130) of these genes are immune related (Table S5). Gene set enrichment analysis (GSEA) revealed top four biological processes significantly enhanced in MAPK-mutated tumors as all immune-related (Figs $3 C$ and S7). Perforin (PRF1), and four granzymes, GZMA (Cytotoxic T-Lymphocyte-Associated Serine Esterase-3), GZMB (Cytotoxic T-Lymphocyte-Associated Serine Esterase-1), GZMH (Cytotoxic Serine Protease C), and GZMK (Tryptase II) were all significantly up-regulated in MAPK-mutated tumors (Fig 3D), indicating remarkably active, likely cytolytic immune responses in situ. Similar findings were consistently noted in HPV-negative MAPK-mutated tumors (Fig S8A).

\section{The only tumors with inherently $\mathrm{CDB}^{+}$T-cell-inflamed immunoactive, cytolytic tumor microenvironments among seven pathway mutants}

Subsequent immune landscape examination across all seven signaling pathways by Tumor Immune Estimation Resource (TIMER) analysis (Li et al, 2016, 2017) reveals for the first time that treatmentnaive MAPK pathway-mutated HNSCC tumors are the only tumors with significant elevation of $\mathrm{CD}^{+}{ }^{+} \mathrm{T}$-cell infiltration within the tumor microenvironment (Fig 4A and B; $P=0.0030$; size of red-outlined bubbles indicating significance level). Most importantly, MAPKmutant tumors have the most remarkable "CD8 ${ }^{+} \mathrm{T}$-cell-inflamed status" along with most significant and concurrent increases in all three major immunoreactive signature scores, including high cytolytic process (CYT) (Rooney et al, 2015), T-effector activity (T-eff) (Bolen et al, 2017), and antitumor IFN- $y$ signature scores ((Ayers et al, 2017)), all evident of active, constitutive cytolytic T-cell activity native to these tumors (Fig $4 \mathrm{~A}-\mathrm{C}$ ). Whereas for JAK/STAT- and PI3Kmutated HNSCC, only $\mathrm{CD} 8^{+} \mathrm{T}$-cells are increased with no obvious concurrent activation of full active CYT, T-eff, and IFN- $y$ signatures, suggestive of potentially weaker $\mathrm{CD} 8^{+} \mathrm{T}$-cell cytolytic activity in situ (Fig $4 A)$. We also noted increases in dendritic cell $(P=0.0052)$ and neutrophil infiltration levels in MAPK-mutant tumors $(P=0.0235$; Figs $4 \mathrm{~A}$ and $B$ and S9). Nonhierarchical clustering demonstrated associations between $\mathrm{CD}^{+}$T-cells with immune activity signature scores and infiltrations of dendritic cells (antigen-presenting cells) and neutrophils, which were discrete from signatures for $\mathrm{B}$ cell, $\mathrm{CD}^{+}{ }^{+} \mathrm{T}$-cell, and macrophage infiltrations (Fig 5A). Overall, these findings support CD8 ${ }^{+}$ T-reactive tumor microenvironments of MAPK-mutant patient tumors. All these findings are consistently noted in HPV-negative MAPKmutated tumors as well (Figs $4 \mathrm{~A}-\mathrm{C}$ and $\mathrm{S} 8 \mathrm{~B}$ ).

Increases of CD8+ T-cell infiltration in HNSCC tumor microenvironment alone are known to independently predict favorable patient survival (Fig 5B) (Hartman et al, 2018). Our in-house MAPK pathwaymutated HNSCC tumors also demonstrated increases in $\mathrm{CD}^{+}{ }^{+}$T-cells (accompanied by dendritic cells and neutrophil infiltrations) by immunohistochemistry, consistent with the TIMER-predicted CD8 ${ }^{+}$T-cellinflamed, immunoactive microenvironments borne by MAPK-mutated HNSCC tumors in TCGA (Figs 5 C and S10). Figs 5C and S10 show

was used for Pt.25 and HSC-4 samples, $50 \mu \mathrm{g}$ of protein lysate was used, whereas for HSC-6 (because of the relatively low endogenous p-RSK levels intrinsic to this cell line), $100 \mu \mathrm{g}$ of protein lysate was loaded for presentation of signal clarity. Bar graphs showing the quantified changes of p-ErbB3(Y1289) levels upon GDC-0994 treatment ( $\mathrm{N} \geq 4$ independent experiments). (D) Negative correlation between p-ErbB3(Y1289) and p-MAPK(T202/Y204) levels in MAPK-mutant HNSCC cell lines based on the published CCLE-proteomic data (Ghandi et al, 2019) and in MAPK-mutated HNSCC patient tumors (allele frequencies [AFs] >40\%) based on TCPA HNSCC RPPA cohort (Li et al, 2013, 2017a). (E) Immunohistochemical staining for p-ErbB3(Y1289) in MAPK-mutated HNSCC patient tumors with high AFs close to $40 \%$ : T40 (MAPK1 p.D321N with AF = $39.1 \%$ ) and T43 (HRAS p.G12S with AF $=37.3 \%$ ) versus T47 and T82 (both are MAPKWT).

Source data are available for this figure. 
A

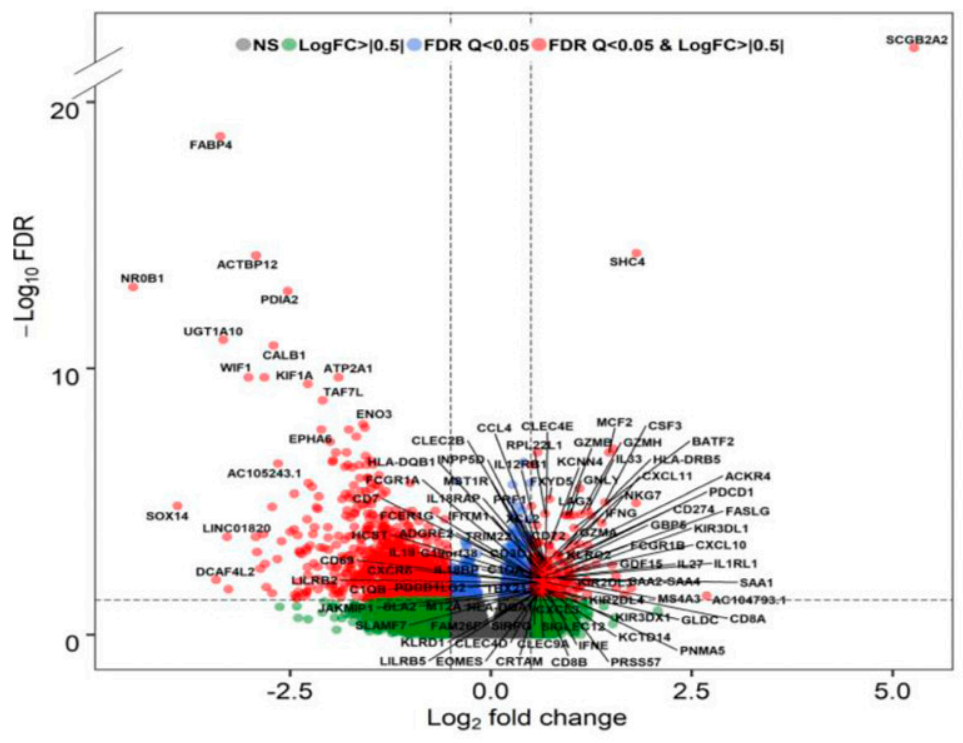

C
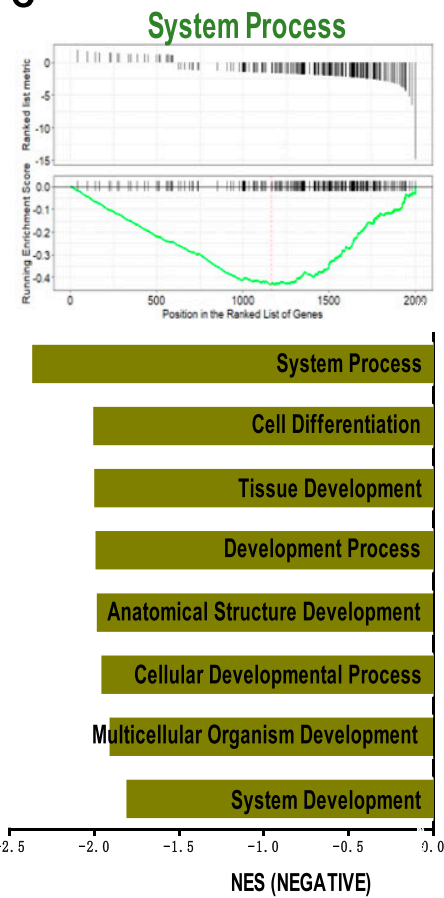

Immune Response
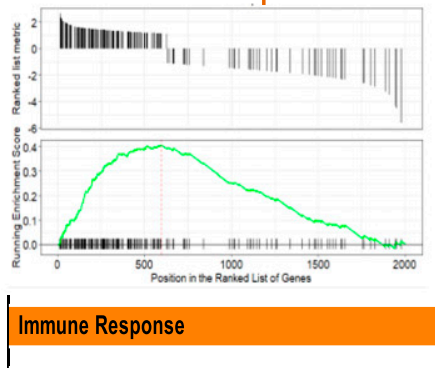

Defense Response

Regulation of Immune System Process

Response to Cytokine

Macromolecule Catabolic Process

Establishment of Protein Localization

Protein Transport

Peptide Transport
B

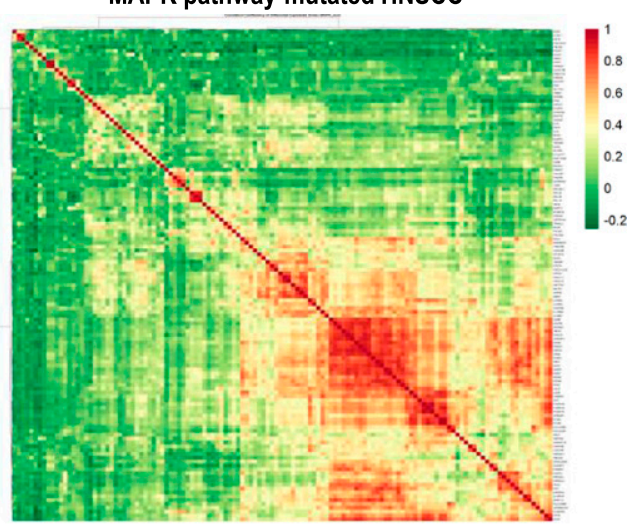

MAPK pathway-WT HNSCC

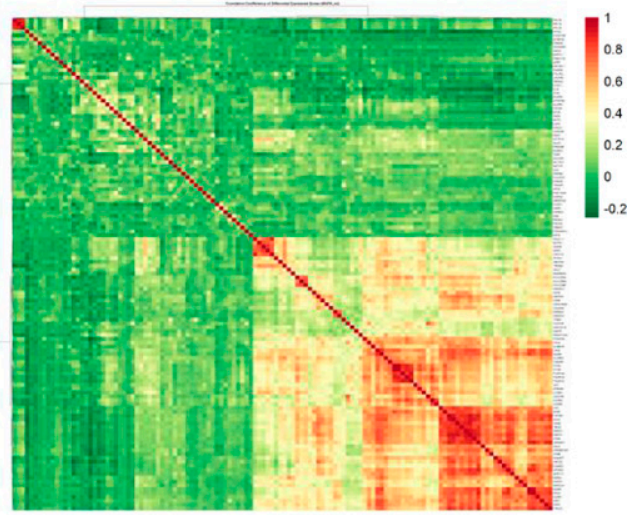

D
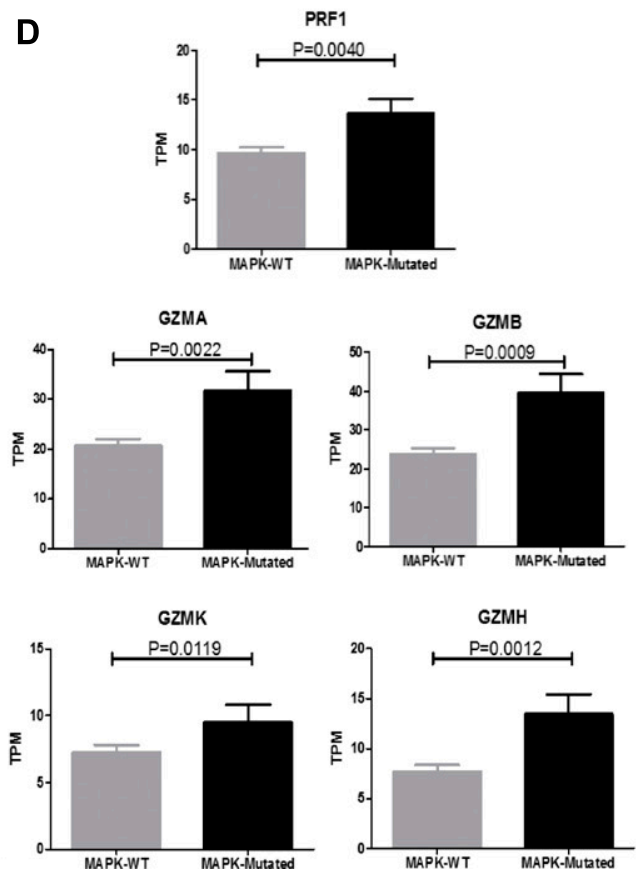

Figure 3. Transcriptomic analyses reveal prominent immune signatures of MAPK-mutant HNSCC tumors.

(A) A volcano plot showing differential RNA expressions between MAPK pathway-mutated versus WT HNSCC tumors. (B) Distinct gene expression patterns of MAPK pathway-mutated tumors versus MAPK-WT tumors (based on RNA-seq dataset of TCGA HNSCC cohort), with 130 protein-coding differentially expressed gene with log 2 fold-change > 0.5 and false discovery rate (FDR) $<0.05$ shown in the respective heat maps. (C) Gene Set Enrichment Analysis for MAPK pathway-mutated (versus MAPK-WT) HNSCC tumors demonstrating enrichment of immune-related gene sets in four of eight enrichment functional gene sets. (D) Comparison of expression levels of PRF1, GZMA, GZMB, GZMH, and GZMK mRNA in MAPK-mutated versus WT HNSCC tumors (based on RNA-seq dataset of TCGA HNSCC provisional cohort). 
A

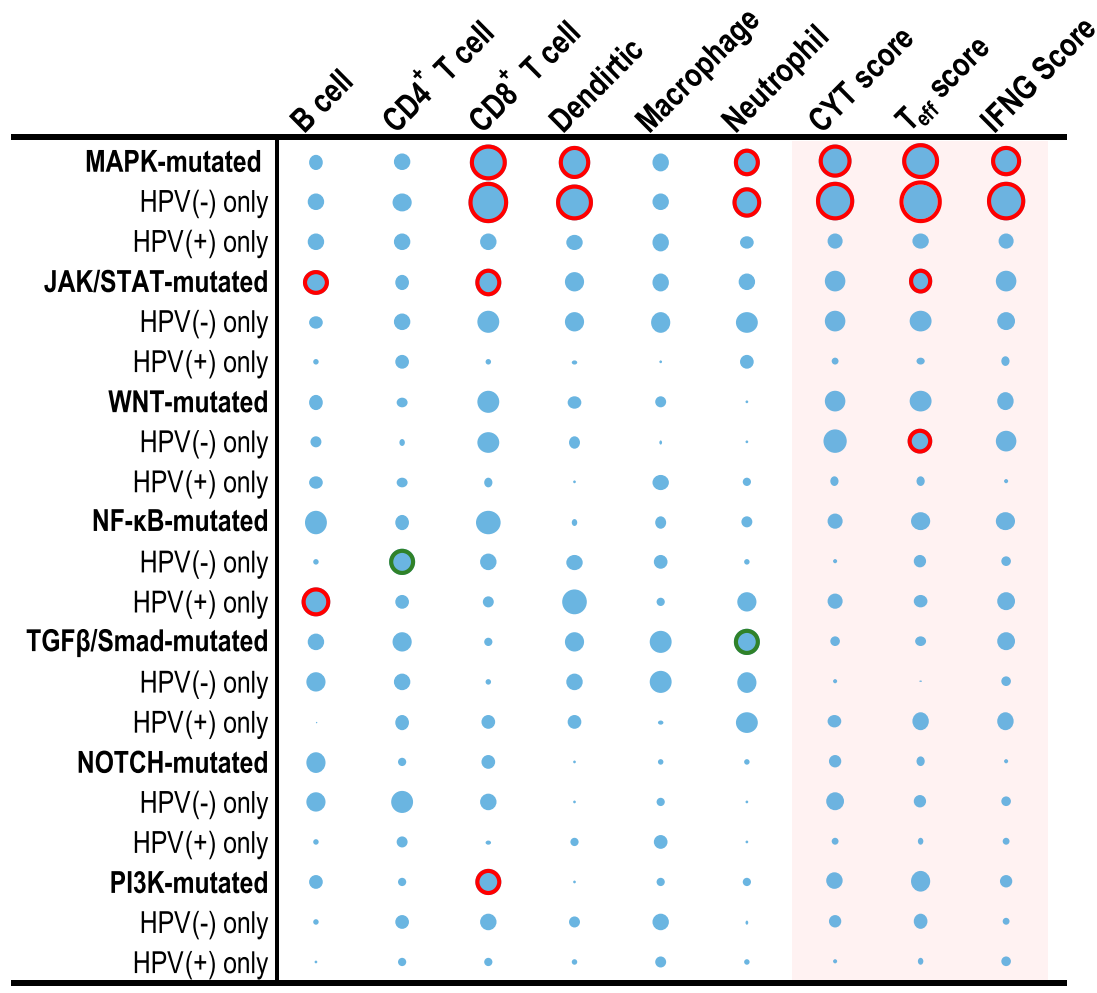

$-\log 10$ (P-value) $\bigcirc$ P-value $<0.05$ and Positive association

P-value $<0.05$ and Negative association

B

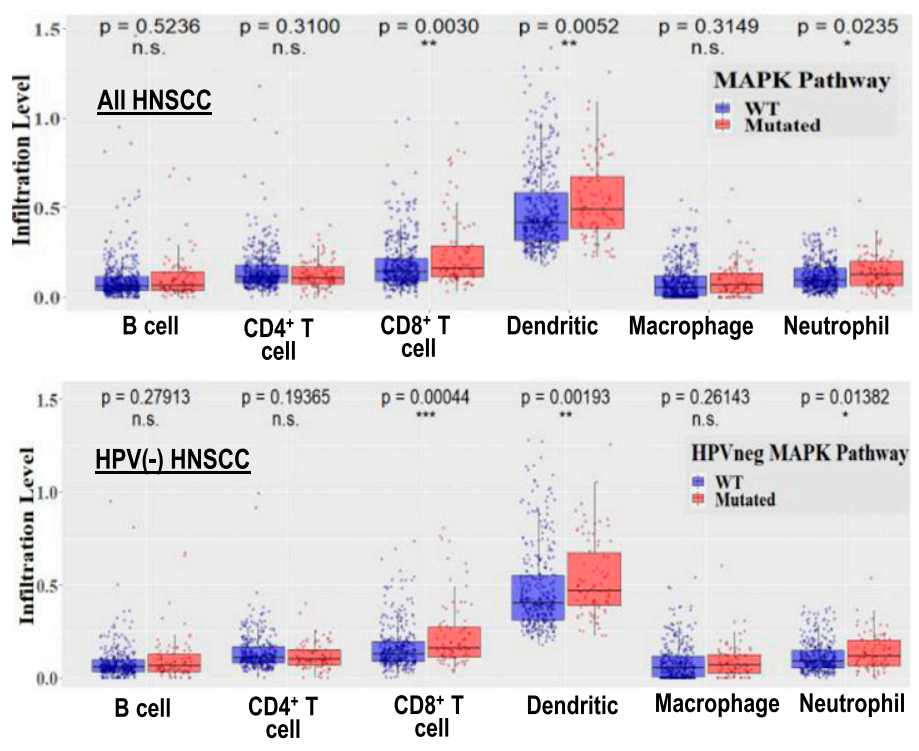

C All HNSCC

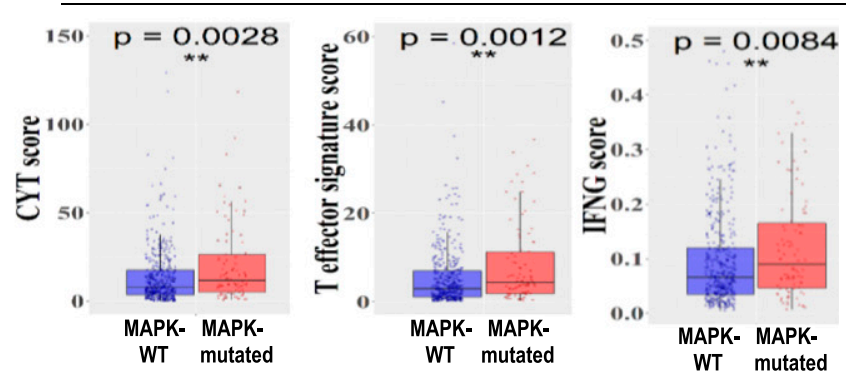

HPV(-) HNSCC

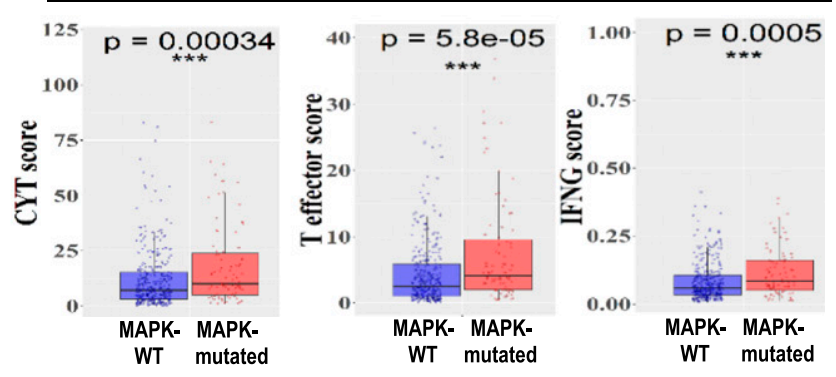

Figure 4. MAPK-mutant HNSCC patient tumors were $\mathrm{CD8}^{+} \mathrm{T}$-cell inflamed with immunoreactive cytolytic signatures.

(A) A bubble plot showing the degree of statistical significance for HNSCC tumor infiltration levels of B cells, $\mathrm{CD} 4^{+} \mathrm{T}$-cells, $\mathrm{CD} 8^{+} \mathrm{T}$-cells, dendritic cells, macrophages, and neutrophils (by TIMER analyses (Li et al, 2016, 2017b), and the CYT score, T-effector score and IFN- $\gamma$ score for HNSCC tumors bearing respective pathway mutations (versus respective WT tumors). Bubbles are highlighted in red outline when $P<0.05$ with calculated positive correlations for increase in the respective TIL or immune score, and bubbles are highlighted in orange outline when $P<0.05$ with calculated negative correlations indicating decrease in the respective TIL or immune score when a pathway is mutated. (B) Results for TIMER analysis for MAPK pathway-mutated versus WT HNSCC tumors for all HNSCC tumors (upper panel), or for human papillomavirus (HPV)-

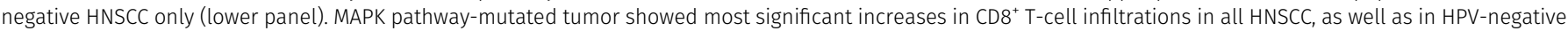
HNSCC (lower panel). P-values were shown for each immune cell type (unpaired $t$ test). (C) Comparisons of CYT score, T-effector signature score, and IFN- $y$ functionality score between MAPK-mutated versus WT HNSCC tumors (for all HNSCC in upper panel; and for HPV-negative HNSCC only in the lower panel). 


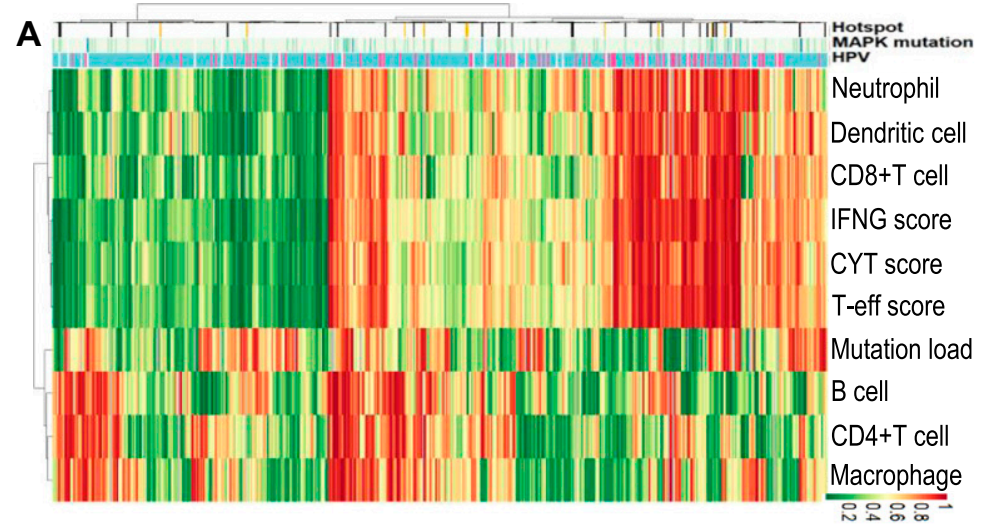

C

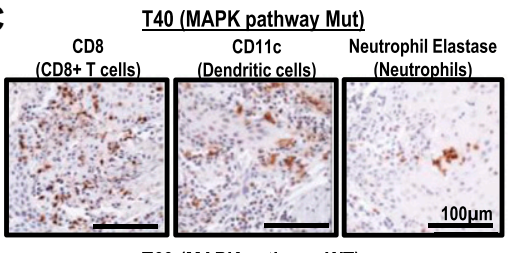

T69 (MAPK pathway WT)

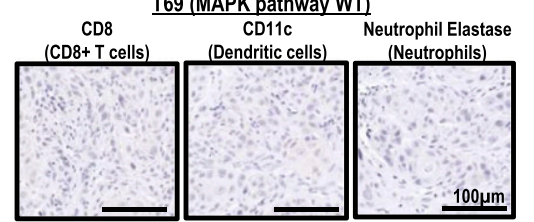

$\mathbf{F}$

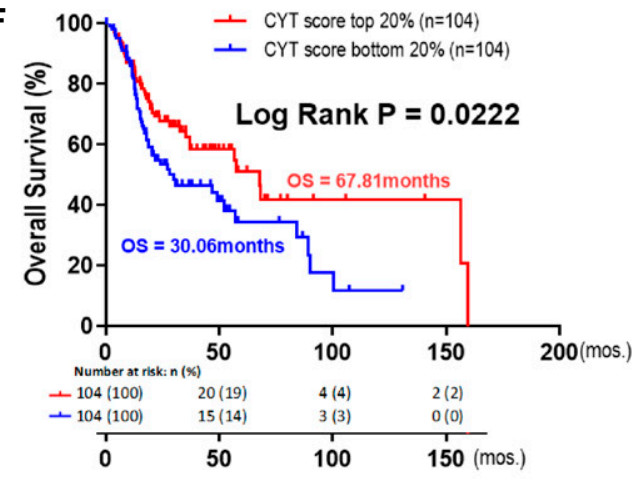

H

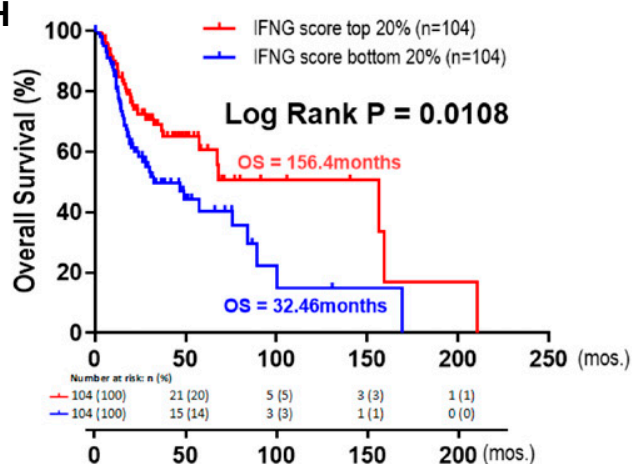

B

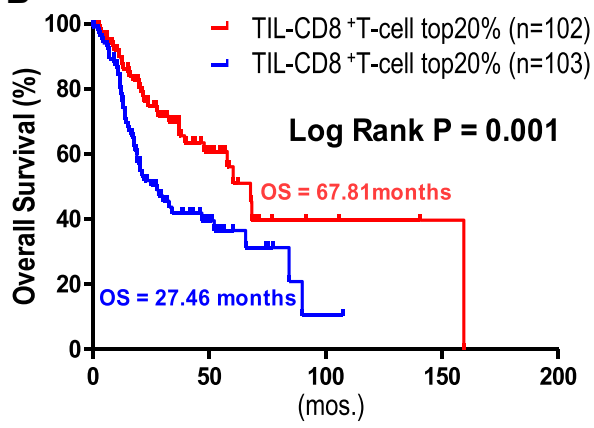

E

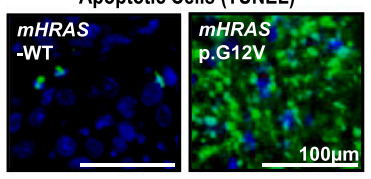

Apoptotic Cells (TUNEL)
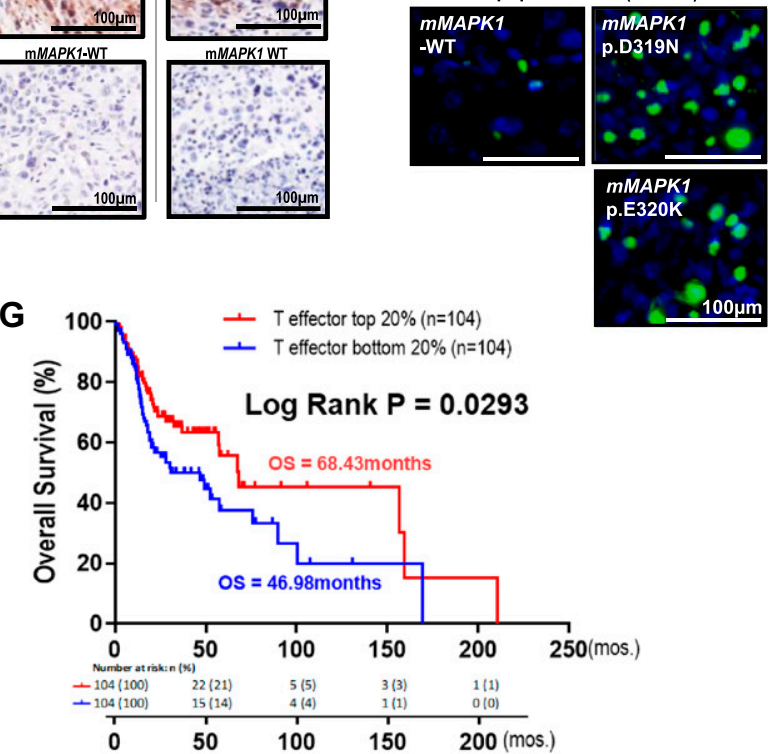

I

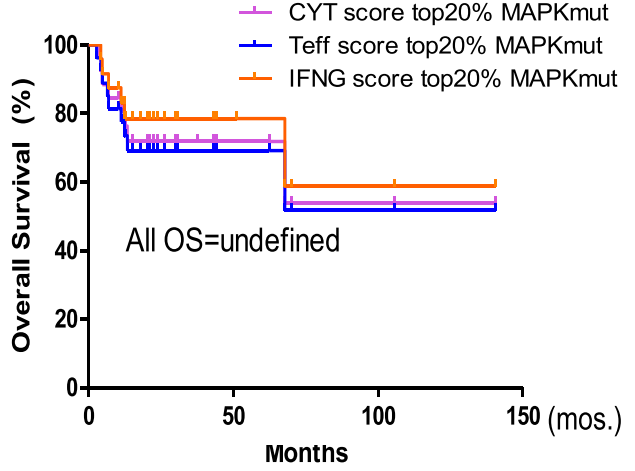

Figure 5. $\mathrm{CD}^{+} \mathrm{T}$-cell-inflamed and cytolytic features of MAPK-mutated head and neck squamous cell carcinoma (HNSCC) were recapitulated in immunocompetent models with massive apoptosis in situ.

(A) Nonhierarchical clustering of tumor infiltrating immune cell types and immune signature scores (CYT, T-effector signature and IFN- $y$ functionality Score) in TCGA HNSCC tumors with MAPK pathway mutational status and HPV status. (B) High infiltrating levels of CD8 ${ }^{+}$T-cells are associated with markedly improved HNSCC patient survival compared with patients with low CD8 ${ }^{+}$T-cell infiltration levels (top and bottom $20 \%$ cutoffs). (C) Immunohistochemical staining of MAPK pathway-mutated HNSCC tumors (T40 with MAPK1 p.D321N hotspot mutation) showing increased expressions of the CD8 marker (for CD $8^{+}$T infiltration). These tumors also expressed higher 
immunohistochemical validation of these heavy immune infiltrations in MAPK-mutant tumors (T40 with MAPK1 p.D321N, T06 with HRAS p.G13D, T25 with HRAS p.G12S, and MAPK1 p.R135K), whereas such infiltrates were not detected in WT tumors (T69, T75, and T39).

\section{Immunocompetent MAPK-mutant HNSCC models display active cell death coupled with massive $\mathrm{CD}^{+}{ }^{+}$-cell recruitment in situ}

As recently recognized, increases in intratumoral $\mathrm{CD}^{+} \mathrm{T}$-cell infiltration in HNSCC dictate patient outcomes (Fig 5B) (Hartman et al, 2018), we determined the ability of MAPK mutations to induce CD $8^{+}$ T-cell infiltration in immunocompetent HNSCC models. Mouse counterparts of three most common human MAPK pathway mutations, and respective WT controls, were expressed into a mouse HNSCC cell line (SCC VII), and their abilities to induce intratumoral CD8 ${ }^{+} \mathrm{T}$-cell infiltration in vivo were compared. As shown in Fig 5D, all three mutants with HNSCC relevance, murine HRAS p.G12V (mHRAS p.G12V), mMAPK1 p.D319N (equivalent to human p.D321N), and mMAPK1 p.E320K mutations (equivalent to human p.E322K), were all potent recruiters for $\mathrm{CD}^{+} \mathrm{T}$-cell infiltration in situ. Furthermore, consistent with our findings that MAPK-mutated human HNSCC tumors have inherently high active cytolytic signatures (Figs 3D and $4 \mathrm{~A}-\mathrm{C}$ ), we also observed remarkable increases in mutant-specific apoptosis in vivo (a two to eight times increase in TUNEL positivity in mutant versus WT tumors; Fig 5E). Our data first established these major MAPK mutations as direct and potent inducers of $\mathrm{CD} 8^{+} \mathrm{T}$ concentration in HNSCC models of isogenic background. Our findings are consistent with an inherently active high cytolytic tumor microenvironment identified in TCGA human MAPK-mutant HNSCC tumors.

\section{Immunoactive and ErbB3-inhibitory activities of MAPK-mutated HNSCC are independent}

In addition to high $\mathrm{CD}^{+}{ }^{+} \mathrm{T}$-cell infiltration, we also identified that very high immune scores, that is, high IFN-y score, high CYT score, and high T-eff signature score (top $20 \%$ versus bottom $20 \%$ arbitrary cutoffs) were all survival-favoring features among HNSCC patients (Fig $5 \mathrm{~F}-\mathrm{H}$; versus patients with tumors of respective low scores). These patients also have reduced chances of death (odds ratios = $0.4949,0.5446$, and 0.5889, respectively, $P<0.05$, Fisher's exact test). Importantly, MAPK pathway-mutated HNSCC patients bearing high IFN-y scores, high CYT scores, and high T-eff scores have long-term survivals (median not reached; Fig 5I). Patients with high immune scores (IFN- $\gamma, \mathrm{CYT}$, and T-eff), defined by extreme cutoffs of quintile, quartile, and tertile (top 20\%, 25\%, and $33 \%$ versus bottom $20 \%, 25 \%$, and $33 \%$, respectively) were almost all significantly associated with better patient survival (except for a demonstrated trend for quartile cutoff with CYT score, $P=0.083$ ). At the most relaxed median cutoff, both the IFN- $y$ and T-eff scores could still separate patient survival $(P<0.05)$, but not CYT score $(P=0.2)$ (Figs S11-S13).

Notably, among MAPK-mutated HNSCC patients, we found that those with high IFN- $y, C Y$, or T-eff scores (both at $20 \%$ and $50 \%$ cutoffs) do not significantly overlap with patients of low p-ErbB3 $(P=n . S$., Fig 6A), suggesting two independent mechanisms operative to contribute the improved outcomes of MAPK pathway-mutant HNSCC. The overall mechanistic findings are summarized in Fig 6B.

\section{MAPK mutations predict immunotherapy outcomes in pan- cancers, oral cancer, and metastatic HNSCC}

Samstein et al (2019) recently published the largest clinical dataset for immune checkpoint inhibitors (ICIS: PD1/PD-L1 and CTLA4 inhibitors) with treatment outcomes in 1,662 advanced or metastatic cancer patients across 11 cancer types. The study concluded that high TMB (high defined as top 20\% patients within each cancer type) could predict $\mathrm{ICI}$ responders in most cancer types (Samstein et al, 2019). Unexpectedly, MAPK pathway mutations alone predict almost two times longer survival in pan-cancer patients upon ICI treatment as compared with MAPK-WT patients (31 versus 16 cutoffs mo, $P<$ 0.0001; Fig 7A). Note that in this target sequencing ICI study, only 10 MAPK members were sequenced. Importantly, $>81 \%$ of pan-cancer patients were TMB-low (Fig 7B). Strikingly, we found that among TMB-low patients, MAPK pathway mutations were also significantly prognostic for favorable survivals with an OS of 25 versus 14 mo in WT patients ( $P=0.0106$, Fig 7C). However, MAPK mutations do not predict survival among TMB-high patients $(P=0.5091)$. Importantly, our novel finding that MAPK pathway mutations could predict favorable outcomes in patients treated with $\mathrm{ICl}$ was further cross-validated in an independent whole-exome sequenced multi-cancer cohort from Miao et al (2018) (Fig 7D).

$\mathrm{CD}^{+}{ }^{+}$-cell infiltration in the tumor microenvironment are believed to be critically involved in the clinical activity of ICls (Melero et al, 2014). As per our finding that MAPK-mutated HNSCC tumors are endowed with inherently high $\mathrm{CD}^{+} \mathrm{T}$-cell-inflamed immunoactive tumor microenvironment with high endogenous cytolytic activity, we analyzed HNSCC patient outcomes from the Samstein-ICI HNSCC dataset. For this HNSCC dataset, oral cancer $(\mathrm{N}=47)$ and oropharyngeal cancer $(\mathrm{N}=32)$ were two major cancers with sufficient case numbers for survival analysis. Strikingly, among advanced oral

levels of CD11c marker (for dendritic cell infiltration) and neutrophil elastase marker (for neutrophil infiltration) when compared with MAPK-WT tumors (T69). Scale bars are shown. (D) Increased expressions of CD8 marker (for CD8 ${ }^{+}$T infiltration) were detected in mHRAS p.G12V mutant, mMAPK1 p.D319N mutant (corresponding to MAPK1 p.D321N mutation in human MAPK1), and mMAPK1 p.E320K mutant (corresponding to MAPK1 p.E322K mutation in human MAPK1), and in their respective mouse WT xenografts by immunohistochemistry on day 6 (for mMAPK1 WT versus mMAPK1 p.E320K pair) and on day 11 (for mHRAS WT versus mHRAS p.G12V \& mMAPK1 WT versus mMAPK1 p.D319N pairs) after tumor cell inoculation. (E) Dramatic increases of apoptotic cells were also observed in MAPK pathway-mutated tumors labeled with TUNEL and corresponding DAPI staining on day 6 after tumor cell inoculation. (F, G, H) Kaplan-Meier overall survival (OS) curves for HNSCC patients with (F) higher IFN- $\gamma$ functionality score, (G) higher T effector signature score, and (H) higher CYT score and versus patients with respective lower IFN- $y$ functionality, T-effector signature and CYT score in TCGA HNSCC ( $N=522$ ) RNA-seq cohort (top $20 \%$ and bottom $20 \%$ cutoffs). (I) Kaplan-Meier OS curves for MAPK pathway mutated patients with high IFN- $\gamma$ functionality score and high T-effector signature score and high CYT score (all top 20\% cutoffs), demonstrating long OS (median OS not reached). 

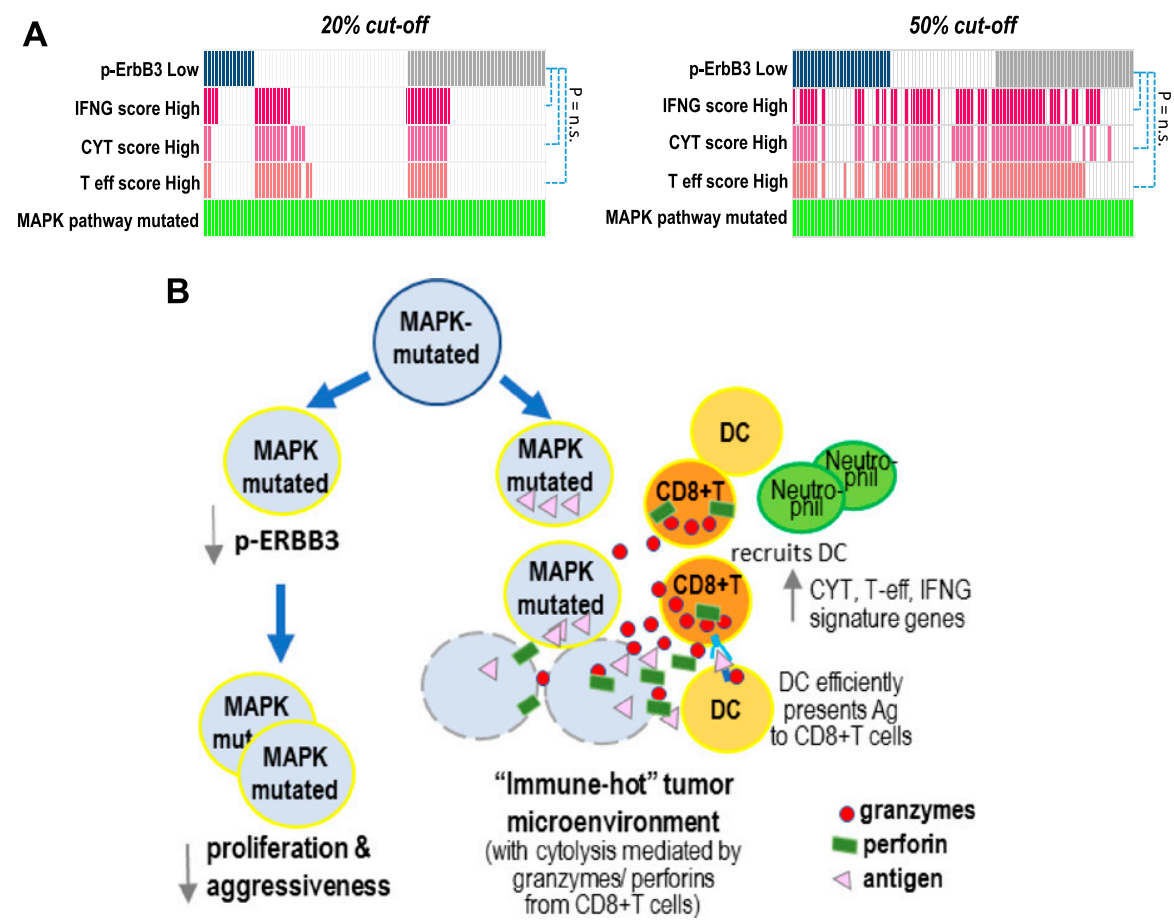

Figure 6. ErbB3 inhibition and $\mathrm{CDB}^{+} \mathrm{T}$-cell immunoactivation as plausible mechanisms contributive to improved survival of MAPK pathway-mutated HNSCC patients.

(A) Oncoprints (20\% and $50 \%$ cutoffs respectively) showing that MAPK pathway-mutated patients with low p-ErbB3 protein expression, and high IFN-y functionality, T-effector signature and CYT score were not significantly overlapping ( $P=$ n.s.). The upper panel shows the

oncoprint with deep blue color ( ) denoting those with bottom $20 \%$ of pErbB3 level (i.e., p-ErbB3 down-

regulation), and levels above that as noncolored white bars ( ), whereas individuals without available RPPA data on p-ErbB3 are denoted by grey bars ( ). Similarly, those with top $20 \%$ immune scores, IFN- $\gamma$ score, CYT score and Teffector are indicated by deep pink (|), light pink (|) and orange ( ), respectively, while non-colored white bars ( ) denote patients with immune scores lower than the top $20 \%$. In the lower panel, the same color coding is adopted, but the colored bars refer to patients with a median (i.e., $50 \%$ cutoffs), that is, lower $50 \%$ for p-ErbB3 level and top 50\% for IFN- $\gamma, \mathrm{CYT}$, and T-effector scores. (B) A schematic summarizing two plausible mechanisms for markedly improved clinical outcomes in HNSCC tumors with MAPK aberrations. cancer patients treated with PD1/PD-L1 inhibitors, MAPK pathway mutations appeared to predict a 3.3 times longer median OS versus WT (33 versus 10 mo, $P=0.0466$; Fig 7E; all tumors with $>10 \%$ tumor purity), supportive of a $\mathrm{CD}^{+} \mathrm{T}$-cell-inflamed phenotype for improved ICl outcome. Whereas TMB-high status only demonstrated a trend for better outcome (Fig S14A), consistent with recent clinical findings that in HNSCC, TMB status may not accurately predict patient outcome as compared with PD-L1 status (Cohen et al, 2019). Importantly, we consistently found that MAPK-mutant oral cancers are significantly associated with immune class defined by Chen et al (2019), with an odds ratio of 0.531 in oral cavity dataset $(N=309, P=0.019$ Fisher's exact test) (Fig 7F). This was not observed in the small oropharyngeal cancer dataset in which no HPV status was recorded (Fig S15).

Notably, as high as $49 \%$ of HNSCC tumors (53/110) sequenced by Samstein et al (2019) were distant metastases (lung, liver, heart, brain, and bone). Strikingly, among this very worst prognostic subgroup with distant metastases, we found that MAPK mutations in metastatic lesions also predicted an approximately four times longer OS versus WT patients upon PD1/PD-L1 inhibitor treatments (median OS of 33 versus 8 mo; Fig 7G). Contrarily, TMB-high status did not predict HNSCC survival (Fig S14B, P = n.s.). Furthermore, in both oral cancer and distant metastatic HNSCC, MAPK-mutant and TMB-high subsets of patients were not overlapping ( $P=$ n.s.; Fig $7 \mathrm{H}$ and I), demonstrating a TMB-independent predictive power of MAPK pathway mutations in HNSCC settings.

Overall, MAPK pathway mutations may represent novel and important HNSCC biomarkers for prognosis in HNSCC. Based on our transcriptome findings, validated in immunocompetent models, MAPK mutations likely identify highly CD8 ${ }^{+}$T-cell-inflamed/cytolytically active HNSCC patients who may potentially benefit from ICls. Our study uncovers novel clinical, biological, and immunological uniqueness of MAPK-mutant HNSCC and may indicate wide clinical utilities of MAPK pathway mutations in this cancer.

\section{Discussion}

We reported for the first time that MAPK pathway mutations have significant survival-favoring clinical, signaling, and immunological impacts in HNSCC. Among seven major cancer-signaling pathways examined, MAPK (ERK) pathway mutations, predominated by many activating mutations, were prognostic for remarkably long patient survivals. This is contradictory to the known role of MAPK-mitogenic signaling in HNSCC tumorigenesis and progression (Gkouveris et al, 2014; Lakshmanachetty et al, 2019). This finding was cross-validated in an independent MSK-IMPACT HNSCC cohort. Furthermore, the prognostic power of MAPK pathway mutations is independent of HPV. Similar superior survival was also observed in MAPK-mutated endometrial cancer patients (TCGA). The finding that MAPK pathway mutations span the diversity of HNSCC subsites (including oral, oropharyngeal, laryngeal, and pharyngeal) to identify patients with good outcomes is superior to that of HPV-positivity (largely restricted to oropharyngeal subsite), and in particular, these mutations predict extraordinary patient outcomes even among TP53-mutated HNSCC patients (median OS $>14 \mathrm{yr}$ ). Thus, MAPK mutations may represent novel prognostic or even de-intensification biomarkers for HNSCC with broad applicability in terms of HNSCC subsites.

Importantly, we also first identified that HNSCC patient tumors with high allele frequencies of MAPK-activating mutations, such as 
A

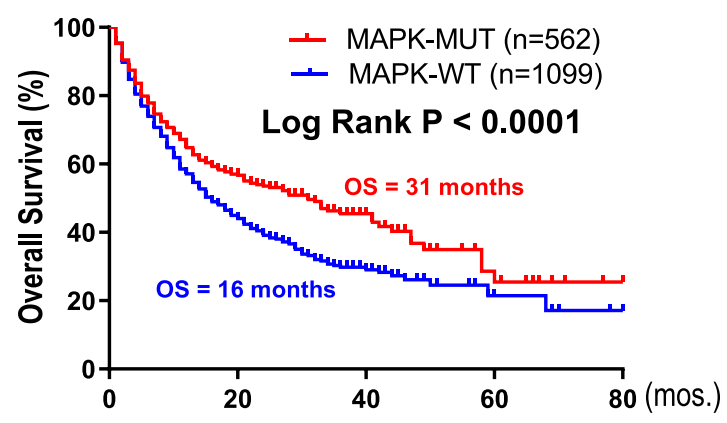

C

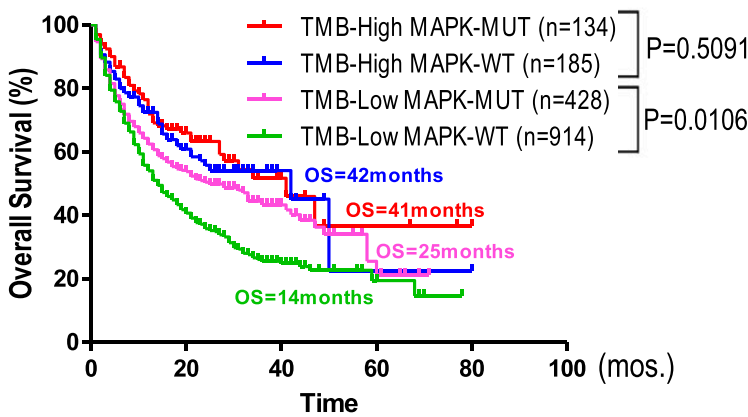

B

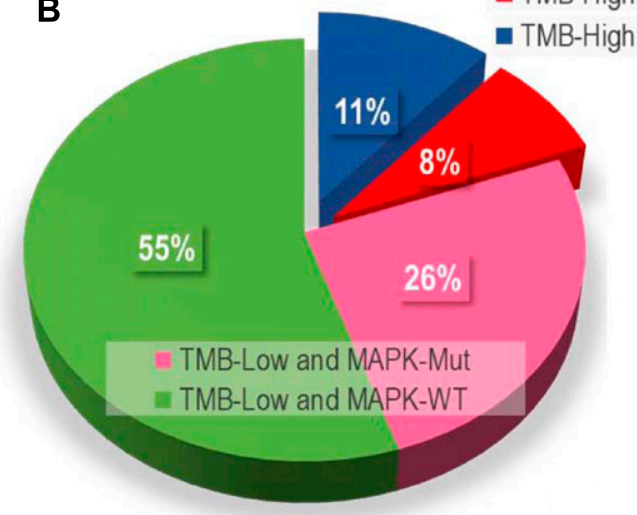

D

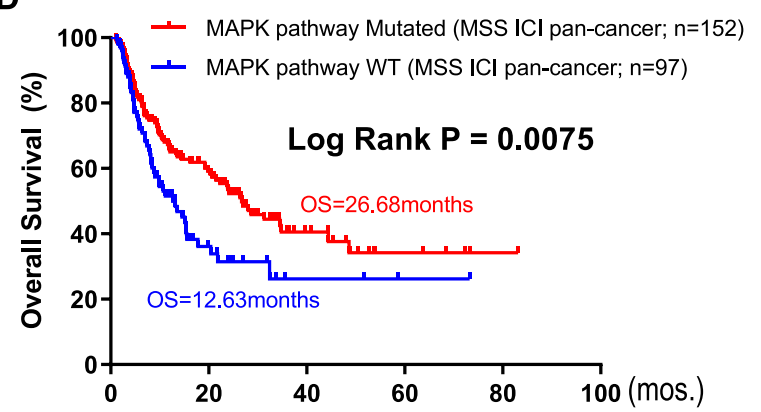

E

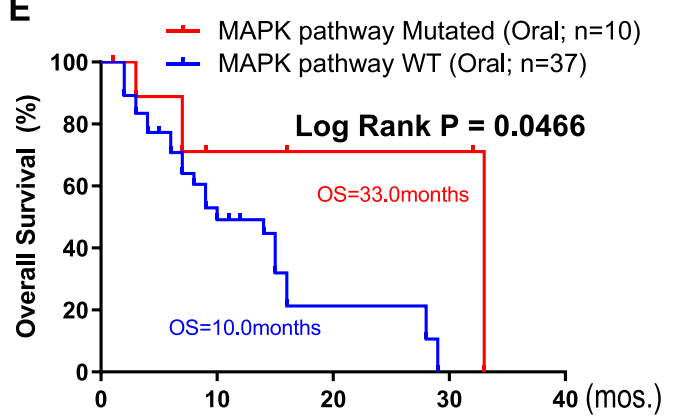

G

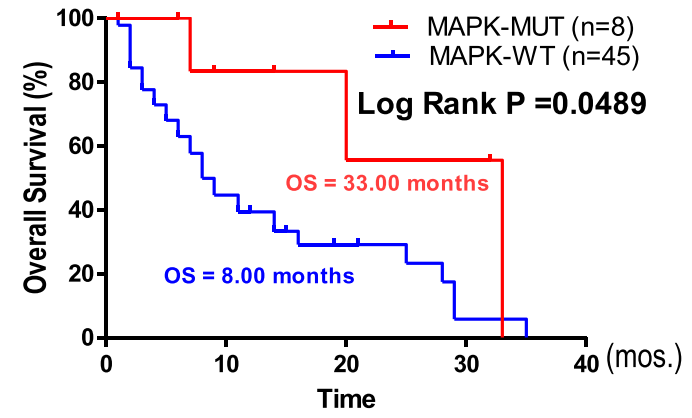

F

\begin{tabular}{c|cc}
\hline & \multicolumn{2}{|c}{ MAPK pathway } \\
Immune Subtype & WT & Mutated \\
\hline Immune Class & 80 & 30 \\
Non-immune Class & 166 & 33 \\
\hline
\end{tabular}

Fisher's Exact Test (one-tailed): $P=0.0195$

$\mathrm{OR}=0.5315$

H

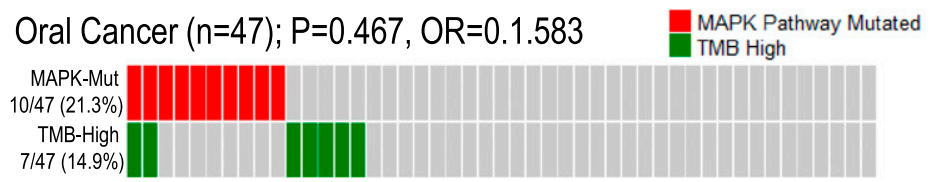

I Metastatic HNSCC $(n=53) ; P=0.527, O R=0.577$ TMB-High 10/53 (18.9\%) MAPK-Mut $8 / 53(15.1 \%)$

Figure 7. MAPK pathway mutations may predict patient outcomes with immune checkpoint inhibitors independent of tumor mutational burden (TMB). (A) MAPK pathway mutations (10 genes) are associated with good clinical outcome from an independent cohort of Samstein et al (2019) in advanced or metastatic pancancer patients $(N=1,662)$ treated with PD1/PD-L1 or CTLA4 inhibitors. (B) A pie chart showing $34 \%$ patients with somatic MAPK pathway mutations in both TMB-high and TMB-low groups of patients in the pan-cancer dataset. High-TMB was previously defined as top $20 \%$ cutoff (i.e., TMB value $\geq 10.3$ for head and neck squamous cell carcinoma [HNSCC]), whereas low-TMB represented the remaining $80 \%$ of patients (i.e., TMB value < 10.3 for HNSCC) per original publication by Samstein et al (2019). (C) Overall survival (OS) curves of four subgroups of patients: TMB-high with MAPK mutations, TMB-high with MAPK-WT, TMB-low with MAPK mutations, and TMB-low with MAPK-WT in 
HRAS and MAPK1 hotspot mutations and potentially others, have remarkable tumoral ErbB3-suppression (first identified in TCGA data and validated in HNSCC tumors). Mechanistically, our findings identified a previously undescribed mechanism of $p$-ErbB3 regulation by MAPK pathway-mutants. Such a negative regulation of p-ErbB3 by ERK activity is uniquely found in MAPK-mutant, but not in MAPK-WT HNSCC. Most importantly, MAPK-mutant HNSCC tumors are the only tumors having significant " $\mathrm{CD} 8^{+}$T-cell-inflamed" and inherently immunoactive tumor microenvironment (versus six other pathway-mutant tumors), with constitutive cytolysis. The ability of MAPK mutations to drive a $\mathrm{CD} 8^{+} \mathrm{T}$-cell-inflamed status in vivo with marked apoptosis is proven in immunocompetent HNSCC models. As low tumoral phospho-ErbB3 levels and elevated CD8 ${ }^{+}$ T-cell infiltrations are recently established events indicative of good patient survivals in HNSCC (Takikita et al, 2011; de Ruiter et al, 2017), our study first defined somatic MAPK pathway mutations as novel genomic events governing two outcome-favoring features in HNSCC. We further showed that the ErbB3-suppressive and $\mathrm{CD}^{+}$ T-cell-inflamed tumor microenvironments of MAPK pathway mutant HNSCC tumors are likely two independent molecular characteristics of MAPK-mutated HNSCC patients with remarkably improved outcomes.

Our findings not only enrich our understanding of the immune uniqueness of MAPK-mutated HNSCC tumors in patients but also highlights the potential clinical utility of MAPK pathway mutations in identifying HNSCC patients with $\mathrm{CD}^{+}$T-cell-inflamed tumors, independent of TMB, for likely beneficial PD1/PD-L1 inhibitor treatments. This positive prognosticity of MAPK pathway mutations may potentially be beneficial to pan-cancer, as shown in two independent immunotherapy cohorts. In conclusion, our study uncovers novel clinical, biological, and immunological understanding of MAPK pathway mutations in HNSCC, which may have important clinical impacts on HNSCC management as prognostic biomarkers and as predictive biomarkers for potential immunotherapy benefits.

\section{Materials and Methods}

\section{Pathway component definitions and databases used}

The MAPK pathway was defined as $H / K / N-R A S, A / B-R A F, R A F 1$, MAP2K1/2 (MEK1/2), MAPK1/3(ERK2/1), RPS6KA1, SHC1/2/3/4, GRB2, and Erk1/2-specific DUSP3/5/6/7/9. The PI3K pathway was defined as AKT1/2/3, PIK3CA/B/D/G/2A/2B/2G, PIK3AP1, PIK3IP1, PDK1, MTOR, TSC1, TSC2, PTEN, RICTOR, RPTOR, RHEB, and PIK3R1/2/3/4/5/6. The NF-KB pathway was defined as TAB1/2/3, MAP3K7/14, CHUK,
IKBKB, IKBKG, NFKBIA, NFKBIE, REL, RELA/B, NFKB1/2, LTBR, TNF, TNFAIP3, TNFSF11/13B, TNFRSF1A/8/11A/13C, BTRC, CYLD, NLRC5, TRADD, CD40, CD40LG, LTA, TRAF2/3/5/6, IL1B, and IL1R1. The JAK/ STAT pathway was defined as JAK1/2/3, STAT1/2/3/4/5A/5B/6, PTPN11, IL6, IL6R, IL6ST, and SOCS3. The Notch pathway was defined as DLL1/3/4, JAG1/2, NOTCH1/2/3/4, NUMB, DTX1/3L, NEDD4, MAML1, RBPJ, POFUT1, HES1/5, and HEY1/2/L. The WNT pathway was defined as WNT1/3A/5A/5B/7A, CTNNB1, HNF1A, FZD1/2/3/7/8/9/10, AXIN1, LEF1, LOXL2, DVL2/3, NKD1/2, TAB1/2, GSK3B, CSNK1A1, NLK, and $L R P 5 / 6$. The TGF- $\beta /$ Smad pathway was defined as SMAD1/2/3/4/5/ 6/7/9, TGFB1/2/3, TGFBR1/2, INHBA/B/C/E, NODAL, ACVR1/1B/1C/ 2A/2B, BMP2/4/7, BMPR1A/B/2, AMHR2, LTBP1, BAMBI, ZFYVE9, SMURF1/2, and LIMK1. All TCGA whole-exome sequencing data and clinical data of TCGA are downloaded from the www.cbioportal.org (Cerami et al, 2012; Gao et al, 2013) on 28 November, 2018. Wholeexome sequencing, RNA-seq, and clinical survival data are available for 510, 522, and 527 (397 for disease-free) HNSCC cases, respectively. Protein quantitative expression levels were downloaded from TCPA level 4 data on 21 June, 2018 (Li et al, 2013, 2017a). Kaplan-Meier survival curves are plotted with GraphPad Prism 5 (USA), with calculated log-rank test $P$-values. The favorable/unfavorable OS indicated in Fig $1 \mathrm{~B}$ and $\mathrm{E}$ are defined by log-rank $P$-value $(P<0.05)$ generated from Kaplan-Meier curves as in Fig $1 C$ and D. In Fig 1B, patients are stratified by the specified status, for example, MAPK pathway mut versus MAPK pathway WT, PI3K pathway mut versus PI3K pathway WT, HPV(+) versus HPV(-), etc. Then, the OS advantages are being analyzed with Kaplan-Meier survival analyses. Only those patient groups with log-rank $P$-value $<0.05$ (significant) would we consider them having favorable/unfavorable OS. CCLEproteomic database was downloaded from DepMap portal (http:/ / www.depmap.org) as published by the CCLE study (Ghandi et al, 2019), and immunotherapy clinical database and targeted sequencing database were downloaded from the studies by Samstein et al (2019) and Miao et al (2018).

\section{Cell cultures and drug treatment}

FaDu cells and HSC-4 cells were purchased from ATCC and JCRB, respectively. Cell lines around passages 10-25 were used (within 6 mo of purchase, which were mycoplasma free when purchased). The Platinum-A (PLAT-A) retrovirus packaging cell line was purchased from Cell Biolabs. The HSC-6 cell line was a generous gift from Dr I Inazawa (Tokyo Medical and Dental University, Japan), and SCC VII mouse HNSCC cell line was kindly provided by Dr Sven Branduau (University Hospital Essen, Germany). Pt-25 primary cultures were prepared from a female recurrent HNSCC patient. For GDC-0994 treatment, the cells were plated at $30 \%$ confluency for

the Samstein study. (D) Kaplan-Meier OS curves for MAPK pathway-mutated HNSCC patients versus MAPK pathway WT patients (MSS ICI pan-cancer cohort; N = 249). (E) Kaplan-Meier OS curves for MAPK pathway-mutated HNSCC patients versus MAPK pathway WT patients (study by Samstein et al (2019); HNSCC Ooal subsite cohort; N = 47). (F) Table of Fisher's exact test showing association of HNSCC subtypes with the immune class as defined by Chen et al (2019). (G) In HNSCC patients with distant metastases (lung, liver, heart, brain, and bone), MAPK pathway mutations are associated with better OS upon PD1/PD-L1 inhibitor treatment ( $P$ = 0.0489; based on databases from Samstein et al (2019)). (H, I) The corresponding oncoprints showing no significant overlap between (H) patients with MAPK pathway mutations and high tumor mutational burden in this HNSCC-oral cancer cohort (TMB score 20\% cutoff within HNSCC histology [N = 139] in the study by Samstein et al (2019)) and (I) no significant overlap for patients with MAPK pathway mutations and high tumor mutational burden in this HNSCC distant metastasis cohort (TMB score $20 \%$ cutoff within HNSCC histology). 
overnight and then subjected to either vehicle or $0.5 \mu \mathrm{M}$ of GDC-0994 for $30 \mathrm{~min}$. The cells were then washed with $1 \times$ PBS, and protein lysates were prepared for Western blot analyses.

\section{Retroviral introduction of pathway genes/mutants into HNSCC models}

The retroviral Plat-A amphotropic expression system (Cell Biolabs, Inc.) was used for ectopic expression of genes and mutants into HNSCC cells. In brief, the desired genes/mutants cloned into the pMXs-puro retroviral expression vector backbone were transfected into PLAT-A cells using Lipofectamine 3000 (Thermo Fisher Scientific) for the generation of retroviruses. Retroviruses were filtered through a $0.45-\mu \mathrm{m}$ mixed cellulose ester membrane filter to remove cell debris and subsequently used for infection of FaDu cells or SCC $\mathrm{VII}$ cells for $48-72 \mathrm{~h}$ at $37^{\circ} \mathrm{C}, 5 \% \mathrm{CO}_{2}$. Retroviruses were removed from the infection medium at postinfection, and cells were then cultured in their respective culture media. Gene expression were then validated by Western blotting. For mouse HNSCC tumor cell inoculation (SCC VII, originally derived from C3He mouse background [Suit et al, 1985]), 2-3 million cells expressing each gene or the respective mutant were subcutaneously injected into females of the $\mathrm{C} 3 \mathrm{H} / \mathrm{He}$ sub-strain (C3HeB/FeJLe-a) mouse strain for tumor establishment with a 26G Hamilton syringe. Tumors were harvested at the designated days for tumor collection. All animal experiments were approved by the University Animal Experimentation Ethics Committee of the Chinese University of Hong Kong.

\section{Western blotting}

Cells were washed with cold 1× PBS and lysed with the NP40 lysis buffer (1\% Nonidet-P40, $150 \mathrm{mM} \mathrm{NaCl} 1 \mathrm{mM}$ EDTA, $10 \mathrm{mM}$ sodium phosphate buffer, and protease phosphatase inhibitor). Cell lysates were centrifuged, and the supernatant was quantified with Protein Assay Dye Reagent (Bio-Rad). 50 mg of protein lysates were mixed with a $4 \times$ protein loading dye and then separated by SDS-PAGE. Separated proteins were transferred to nitrocellulose membrane, which was then blocked with $5 \%$ nonfat dry milk (in TBST; $150 \mathrm{mM}$ $\mathrm{NaCl}, 50 \mathrm{mM}$ Tris, and 0.1\% Tween 20, pH 7.4) and probed with primary antibody at $4^{\circ} \mathrm{C}$ overnight. Primary antibodies include AKT (\#9272), pi-AKT (\#9271), ARAF(\#4432), pi-ARAF(S299) (\#4431), BRAF (\#2696), pi-BRAF(S445) (\#2696), pi-ErbB3 (\#2842), ErbB3 (\#12708), MAPK (\#9102), pi-MAPK (\#9101), pi-MEK1/2 (\#9154), and RSK1 (\#8408), all from Cell Signaling Technology, USA. Anti- $\beta$-actin (sc-69879) antibody was from Santa Cruz. Anti-MEK1/2 (YT2714) and anti-N/ H/K-RAS (YT2960) antibodies were from ImmunoWay. The probed membrane was then washed three times with $1 \times$ TBST, followed by a 1-2-h incubation with the respective secondary antibody (Goat anti-Mouse [ab97230; Abcam] and HRP-Goat anti-Rabbit [65-6120; Invitrogen]), followed by $3 \times$ washings with $1 \times$ TBST. ECL detection solution was then applied onto the membrane for the development of chemiluminescence, which was captured by autoradiography.

\section{TIMER analysis for immune infiltrates}

We adopted the methodology of TIMER analysis for immune infiltration level estimation (Li et al, 2016, 2017b). The infiltration level of six immune cell types (B cell, $\mathrm{CD}^{+} \mathrm{T}$-cell, $\mathrm{CD}^{+} \mathrm{T}$-cell, macrophage, neutrophil, and dendritic cell) in 512 TCGA-HNSCC head and neck squamous carcinoma tumor samples are extracted from the TIMER Web site (https:// cistrome.shinyapps.io/ timer/_W_20aca96c/immuneEstimation.txt) and grouped according to the mutational information downloaded from cBioPortal (https://www.cbioportal.org/study/summary?id=hnsc_tcga). The Wilcoxon rank sum test was performed to calculate the statistical significance between two groups in the TIMER plots.

\section{RNA-seq and GSEA}

TCGA HNSCC RNA-seq data were downloaded from the NCI Genomic Data Commons portal (https://portal.gdc.cancer.gov/repository? facetTab=files\&filters=\%7B\%22op\%22\%3A\%22and\%22\%2C\%22content \%22\%3A\%5B\%7B\%22op\%22\%3A\%22in\%22\%2C\%22content\%22\%3A\%7B \%22field\%22\%3A\%22cases.project.project_id\%22\%2C\%22value\%22\% 3A\%5B\%22TCGA-HNSC\%22\%5D\%7D\%7D\%2C\%7B\%22op\%22\%3A\%22in \%22\%2C\%22content\%22\%3A\%7B\%22field\%22\%3A\%22files.data_category \%22\%2C\%22value\%22\%3A\%5B\%22Transcriptome\%20Profiling\%22\% 5D\%7D\%7D\%5D\%7D\&searchTableTab=files) (Grossman et al, 2016) using GDCRNATools (R package). Differential gene expression analysis between MAPK-mutated and MAPK-WT samples was performed using the method of DESeq2. Pearson correlation coefficients among 130 DEGs were calculated for the plotting of the correlation heatmap with pheatmap ( $\mathrm{R}$ package) using the wardD clustering method. GSEA was run with the clusterProfiler (R package).

\section{Cytolytic score, T-effector signature score, and antitumor IFN- $y$ score calculations}

Cytolytic score (CYT) was calculated by the geometric mean of the TPM of GZMA and PRF1 as previous described by Rooney et al (2015) (offset 0.01) (Rooney et al, 2015). Similarly, based on the T-effector signature gene list, T-effector (T-eff) score was calculated as the geometric mean of the TPM of GZMA, GZMB, PRF1, IFN- $\gamma$, EOMES, and CD8A (offset 0.01) ((Bolen et al, 2017)). Antitumor IFN- $y$ score was the weighted arithmetic mean of the TPM of 18 IFN- $\gamma$ expanded immune gene signatures as previously described by (Ayers et al, 2017). These include CD3D, IDO1, CIITA, CD3E, CCL5, GZMK, CD2, HLA-DRA, CXCL13, IL2RG, NKG7, HLA-E, CXCR6, LAG3, TAGAP, CXCL10, STAT1, and GZMB.

\section{Tumor samples and targeted sequencing}

Tumor tissues and blood samples were collected from patients under written informed consents according to clinical research approvals by the Institutional Review Board of the University of Hong Kong/Hospital Authority Hong Kong East Cluster Research Ethics Committee (for Queen Mary Hospital), the Joint Chinese University of Hong Kong-New Territories East Cluster Clinical Research Ethics Committee (for Prince of Wales Hospital), Hong Kong SAR, and the Research Ethics Committee, Kowloon West Cluster (for Yan Chai Hospital), Hong Kong SAR. Genomic DNA from samples were extracted with the DNeasy Blood \& Tissue Kit (QIAGEN), followed by quantification and targeted sequencing by next-generation sequencing using the IonS5 platform (Thermo Fisher Scientific). All samples were sequenced with a mean depth of $>500 \times$ using a 
Table showing the sequenced locations and the aminoacids convered of the partially sequenced genes in our custom-designed MAPK pathway gene panel.

\begin{tabular}{|c|c|c|c|}
\hline Gene & Chromosome & Locus position & Amino acid position \\
\hline ARAF & ChrX & $47426003-47426252$ & $186-233$ \\
\hline$B R A F$ & Chr7 & $\begin{array}{l}140434532-140434640,140439596-140439815,140449044- \\
140449156,140449186-140449273,140453021-140453215 \\
140453948-140454071,140476652-140476772,140476828- \\
140476946,140477788-140477937,140481362-140481448\end{array}$ & $454-526,545-631,642-667,679-722$ \\
\hline HRAS & Chr11 & $533782-533882,534220-534306$ & $6-34,58-91$ \\
\hline KRAS & Chr12 & $\begin{array}{l}\text { 25368427-25368555, 25378527-25378605, 25378635-25378758, } \\
25380260-25380368,25398189-25398310\end{array}$ & $4-66,98-121,132-173$ \\
\hline MAPK1 & Chr22 & $\begin{array}{l}\text { 22123469-22123664, 22127153-22127280, 22142514-22142695, } \\
\text { 22142879-22143107, 22153230-22153505, 22160105-22160333, } \\
\text { 22161922-22162142, 22221511-22221644 }\end{array}$ & $29-362$ \\
\hline MAP2K1 & Chr15 & $\begin{array}{l}\text { 6667947-66679845, 66727308-66727523, 66727541-66727651, } \\
66728983-66729286,66735605-66735722,66736897- \\
66737080,66774071-66774222,66777239-66777548,66779529- \\
66779647,66781535-66781633,66782015-66782132,66782829- \\
66782961\end{array}$ & $21-356$ \\
\hline MAP2K2 & Chr19 & $\begin{array}{l}\text { 4090254-4090351, 4090679-4090789, 4094494-4094602, } \\
\text { 4095288-4095521, 4097192-4097295, 4097316-4097449, } \\
\text { 4099378-4099495, 4101127-4101237, 4101257-4101387, } \\
\text { 4102338-4102451, 4110387-4110512, 4110586-4110696, } \\
\text { 4117321-4117447, 4117464-4117566 }\end{array}$ & $\begin{array}{l}52-85,92-124,149-183,191-198,236-246,308-315,323-350,365- \\
373,483-493\end{array}$ \\
\hline
\end{tabular}

custom-designed gene panel on major MAPK pathway genes. The custom gene panel consisted of amplicons that covered all exons of MAP2K1 and MAPK1. It also captured selection regions of ARAF, BRAF, HRAS, KRAS, and MAP2K2 to cover all well-known hotspot sites. The sequenced locations for these partially sequenced genes are listed in the following table, and variants were called by the Ion Reporter Software (Thermo Fisher Scientific).

\section{Immunohistochemistry and TUNEL assay}

Patient tumors were freshly fixed with $10 \%$ formalin and dehydrated in a serial manner in ethanol. The formalin fixed and paraffin embedded tumor samples were then sectioned and dewaxed and rehydrated in xylene, $100 \%$ ethanol, $70 \%$ ethanol, and running tap water. Antigen retrieval was performed at $95^{\circ} \mathrm{C}$ for $20 \mathrm{~min}$ in citrate buffer ( $10 \mu \mathrm{M}$ citrate acid and $0.05 \%$ Tween $20, \mathrm{pH} 6.0$ ). The VECTASTAIN Elite ABC Universal PLUS Kit Peroxidase (Horse AntiMouse/Rabbit IgG) (Cat. no. PK-8200) was used for immunohistochemical staining. Endogenous peroxidase activity was quenched by BLOXALL Blocking Solution, followed by blocking in 2.5\% Normal Horse Serum for $20 \mathrm{~min}$ at room temperature. CD8 mouse antihuman antibody (Cat. no. Ab17147, 1:500; Abcam), CD11c rabbit antihuman antibody (Cat. no. Ab52632, 1:500; Abcam), and neutrophil elastase rabbit antihuman antibody (Cat. no. Ab68672, 1:500; Abcam) and phospho-HER3/ErbB3(Tyr1289) (21D3) rabbit antihuman antibody (\#4791, 1:100; Cell Signaling Technology) were used as primary antibodies to stain patient tumors for overnight incubation at $4^{\circ} \mathrm{C}$. CD8 rabbit antimouse antibody (Cat. no. 203035, 1:750; Abcam) and cytokeratin mouse antibody (Cat. no. M3515, 1:500; DAKO) were used. The secondary antibody (prediluted biotinylated horse antimouse/ rabbit IgG secondary antibody) was added for 1-h incubation at room temperature. For signal amplification and detection, the slides were incubated with VECTASTAIN Elite ABC reagent for 30 min and ImmPACT DAB EqV solutions for $30 \mathrm{~s}$. After counterstaining with hematoxylin for $1 \mathrm{~min}$, clearing, and mounting, pictures were taken under a light microscope. The Roche In Situ Cell Death Detection Kit, Fluorescein (Cat. no. 11684795910) was applied for TUNEL assay. Proteinase K solution (Mat No. 1014023; QIAGEN) was used to pretreat the rehydrated sections. Then the TUNEL reaction mixture was incubated on the section in dark at $37^{\circ} \mathrm{C}$ for $1 \mathrm{~h}$ in dark. The section was mounted with VECTASHIELD vibrance antifade mounting medium (Cat. no. H-1800).

\section{Supplementary Information}

Supplementary Information is available at https://doi.org/10.26508/lsa. 201900545

\section{Acknowledgements}

This research is funded by the General Research Fund (\#17114814 to VWY Lui, Research Grant Council, Hong Kong) and VWY Lui also receives funding from General Research Fund, Research Grant Council, Hong Kong government, Hong Kong SAR (\#17121616, \#14168517), Research Impact Fund (\#R4017-18), the Health and Medical Research Fund (HMRF\#15160691, the Health and Medical Research Fund, the Food and Health Bureau, the Government of the Hong Kong Special Administrative Region), University-Industry Collaboration Program (UIM/329; Innovation and Technology Fund, Hong Kong government, Hong Kong SAR), and the Hong Kong Cancer Fund, Hong Kong SAR. Y Liu and W Piao receive funding supports (Postdoctoral Hub PH-ITF Ref.: PiH/052/18 and $\mathrm{PiH} / 234 / 18$ of UIM/329) from the Innovation and Technology Fund, Hong Kong 
government. JYK Chan receives funding support by the Dr Stanley Ho Medical Foundation and the General Research Fund (\#14109716; \#14108818 General Research Fund, Research Grant Council, Hong Kong government, Hong Kong SAR). YX Su receives funding support from Hong Kong Research Grant Council-General Research Fund \#17120718. JR Grandis receives funding from National Institudes of Health grants R35CA231998, U54CA209891, R01DE023685, and R01DE028289.

\section{Authors Contributions}

H-L Ngan: conceptualization, data curation, formal analysis, validation, investigation, methodology, and writing-original draft, review, and editing.

Y Liu: conceptualization, data curation, software, formal analysis, validation, investigation, methodology, and writing-original draft, review, and editing.

AY Fong: data curation, formal analysis, validation, and investigation. PHY Poon: data curation, formal analysis, validation, and investigation. CK Yeung: data curation, formal analysis, validation, and investigation. SSM Chan: data curation, formal analysis, supervision, validation, and investigation.

A Lau: data curation, software, formal analysis, validation, investigation, and methodology.

W Piao: data curation, formal analysis, validation, and investigation. H Li: data curation, formal analysis, validation, and investigation.

JSW Tse: data curation, formal analysis, validation, and investigation. K-W Lo: writing-review and editing.

SM Chan: data curation, formal analysis, validation, and investigation. Y-X Su: resources, data curation, and project administration. JYK Chan: resources, data curation, and project administration. CW Lau: resources, data curation, and project administration. GB Mills: writing-review and editing.

JR Grandis: writing-review and editing.

VWY Lui: conceptualization, resources, formal analysis, supervision, funding acquisition, investigation, methodology, project administration, and writing-original draft, review, and editing.

\section{Conflict of Interest Statement}

VWY Lui received a University-Industry Collaboration Program (UIM/329; from the Innovation and Technology Fund, Hong Kong government, and Lee's Pharmaceutical [Hong Kong Limited] in 2018-2020) and served as a scientific consultant for Novartis Pharmaceutical (Hong Kong) Limited (Oct 2015-Oct 2016). JYK Chan served as a consultant for Intuitive Surgical Inc. (Sunnyvale, CA) and advisor for Aptorum Group Ltd. (Hong Kong). JR Grandis is a co-inventor of a cyclic STAT3 decoy and has financial interests in STAT3 Therapeutics, Inc. GB Mills served as a consultant for AstraZeneca, Chrysallis Biotechnology, ImmunoMET, Ionis, Lilly, PDX Pharmaceuticals, Signalchem Lifesciences, Symphogen, Tarveda and Zentalis. GB Mills also has a financial relationship with Catena Pharmaceuticals, ImmunoMet, SignalChem and Tarveda and is holding licensed technologies including HRD assay to Myriad Genetics and DSP patents with Nanostring. Research of GB Mills is sponsored by Nanostring Center of Excellence and Ionis (Provision of tool compounds).

\section{References}

Ayers M, Lunceford J, Nebozhyn M, Murphy E, Loboda A, Kaufman DR, Albright A, Cheng JD, Kang SP, Shankaran V, et al (2017) IFN-gamma-related mRNA profile predicts clinical response to PD-1 blockade. J Clin Invest 127: 2930-2940. doi:10.1172/jci91190
Bolen CR, Mccord R, Huet S, Frampton GM, Bourgon R, Jardin F, Dartigues P, Punnoose EA, Szafer-Glusman E, Xerri L, et al (2017) Mutation load and an effector T-cell gene signature may distinguish immunologically distinct and clinically relevant lymphoma subsets. Blood Adv 1: 1884-1890. doi:10.1182/bloodadvances.2016000786

Cadley J, Simpson D, Ferguson R, Pandya A, Hekal T, Richards T (2018) Mutation burden in conjunction with MAPK-pathway mutation status as a prognostic biomarker of overall melanoma survival. J Clin Oncol 36: 9584. doi:10.1200/jco.2018.36.15_suppl.9584

Cerami E, Gao J, Dogrusoz U, Gross BE, Sumer SO, Aksoy BA, Jacobsen A, Byrne C), Heuer ML, Larsson E, et al (2012) The cBio cancer genomics portal: An open platform for exploring multidimensional cancer genomics data. Cancer Discov 2: 401-404. doi:10.1158/2159-8290.cd-12-0095

Chen YP, Wang YQ, LV JW, Li YQ, Chua MLK, Le QT, Lee N, Colevas AD, Seiwert T, Hayes DN, et al (2019) Identification and validation of novel microenvironment-based immune molecular subgroups of head and neck squamous cell carcinoma: Implications for immunotherapy. Ann Oncol 30: 68-75. doi:10.1093/annonc/mdy470

Cohen EEW, Bell RB, Bifulco CB, Burtness B, Gillison ML, Harrington KJ, Le QT, Lee NY, Leidner R, Lewis RL, et al (2019) The Society for Immunotherapy of Cancer consensus statement on immunotherapy for the treatment of squamous cell carcinoma of the head and neck (HNSCC). J Immunother Cancer 7: 184. doi:10.1186/s40425-019-0662-5

De Ruiter EJ, Ooft ML, Devriese LA, Willems SM (2017) The prognostic role of tumor infiltrating T-lymphocytes in squamous cell carcinoma of the head and neck: A systematic review and meta-analysis. Oncoimmunology 6: e1356148. doi:10.1080/2162402x.2017.1356148

Fakhry C, Gillison ML (2006) Clinical implications of human papillomavirus in head and neck cancers. J Clin Oncol 24: 2606-2611. doi:10.1200/ jco.2006.06.1291

Gao J, Aksoy BA, Dogrusoz U, Dresdner G, Gross B, Sumer SO, Sun Y, Jacobsen A, Sinha R, Larsson E, et al (2013) Integrative analysis of complex cancer genomics and clinical profiles using the cBioPortal. Sci Signal 6: pl1. doi:10.1126/scisignal.2004088

Ghandi M, Huang FW, Jane-Valbuena J, Kryukov GV, Lo CC, Mcdonald ER 3rd, Barretina J, Gelfand ET, Bielski CM, Li H, et al (2019) Next-generation characterization of the cancer cell line encyclopedia. Nature 569: 503-508. doi:10.1038/s41586-019-1186-3

Gillison ML, Trotti AM, Harris J, Eisbruch A, Harari PM, Adelstein DJ, Sturgis EM, Burtness B, Ridge JA, Ringash J, et al (2019) Radiotherapy plus cetuximab or cisplatin in human papillomavirus-positive oropharyngeal cancer (NRG oncology RTOG 1016): A randomised, multicentre, non-inferiority trial. Lancet 393: 40-50. doi:10.1016/s01406736(18)32779-x

Gkouveris I, Nikitakis N, Karanikou M, Rassidakis G, Sklavounou A (2014) Erk1/ 2 activation and modulation of STAT3 signaling in oral cancer. Oncol Rep 32: 2175-2182. doi:10.3892/or.2014.3440

Grossman RL, Heath AP, Ferretti V, Varmus HE, Lowy DR, Kibbe WA, Staudt LM (2016) Toward a shared vision for cancer genomic data. N Engl J Med 375: 1109-1112. doi:10.1056/nejmp1607591

Hartman DJ, Ahmad F, Ferris RL, Rimm DL, Pantanowitz L (2018) Utility of CD8 score by automated quantitative image analysis in head and neck squamous cell carcinoma. Oral Oncol 86: 278-287. doi:10.1016/ j.oraloncology.2018.10.005

Hedberg ML, Peyser ND, Bauman JE, Gooding WE, Li H, Bhola NE, Zhu TR, Zeng Y, Brand TM, Kim MO, et al (2019) Use of nonsteroidal antiinflammatory drugs predicts improved patient survival for PIK3CAaltered head and neck cancer. J Exp Med 216: 419-427. doi:10.1084/ jem.20181936

Lakshmanachetty S, Balaiya V, High WA, Koster MI (2019) Loss of TP63 promotes the metastasis of head and neck squamous cell carcinoma by activating MAPK and STAT3 signaling. Mol Cancer Res 17: 1279-1293. doi:10.1158/1541-7786.mcr-18-1355 
Li B, Severson E, Pignon JC, Zhao H, Li T, Novak J, Jiang P, Shen H, Aster JC, Rodig S, et al (2016) Comprehensive analyses of tumor immunity: Implications for cancer immunotherapy. Genome Biol 17: 174 doi:10.1186/s13059-016-1028-7

Li J, Akbani R, Zhao W, Lu Y, Weinstein J, Mills G B, Liang H (2017a) Explore, visualize, and analyze functional cancer proteomic data using the Cancer Proteome Atlas. Cancer Res 77: e51-e54. doi:10.1158/00085472. CAN-17-0369

Li J, Lu Y, Akbani R, Ju Z, Roebuck P, Liu W, Yang J-Y, Broom B, Verhaak R, Kane D, et al (2013) TCPA: A resource for cancer functional proteomics data. Nat Methods 10: 1046-1047. doi:10.1038/nmeth.2650

Li T, Fan J, Wang B, Traugh N, Chen Q, Liu JS, Li B, Liu XS (2017b) TIMER: A web server for comprehensive analysis of tumor-infiltrating immune cells. Cancer Res 77: e108-e110. doi:10.1158/0008-5472.can-17-0307

Liu YF, Chiang SL, Lin CY, Chang JG, Chung CM, Ko AM, Lin YZ, Lee CH, Lee KW, Chen MK, et al (2016) Somatic mutations and genetic variants of NOTCH1 in head and neck squamous cell carcinoma occurrence and development. Sci Rep 6: 24014. doi:10.1038/srep28409

Lui VW, Hedberg ML, Li H, Vangara BS, Pendleton K, Zeng Y, Lu Y, Zhang Q, Du Y, Gilbert BR, et al (2013) Frequent mutation of the PI3K pathway in head and neck cancer defines predictive biomarkers. Cancer Discov 3: 761-769. doi:10.1158/2159-8290.cd-13-0103

Mehanna H, Robinson M, Hartley A, Kong A, Foran B, Fulton-Lieuw T, Dalby M, Mistry P, Sen M, O'Toole L, et al (2019) Radiotherapy plus cisplatin or cetuximab in low-risk human papillomavirus-positive oropharyngeal cancer (De-ESCALaTE HPV): An open-label randomised controlled phase 3 trial. Lancet 393: 51-60. doi:10.1016/s0140-6736(18)32752-1

Melero I, Rouzaut A, Motz GT, Coukos G (2014) T-cell and NK-cell infiltration into solid tumors: A key limiting factor for efficacious cancer immunotherapy. Cancer Discov 4: 522-526. doi:10.1158/2159-8290.cd13-0985

Miao D, Margolis CA, Vokes NI, Liu D, Taylor-Weiner A, Wankowicz SM, Adeegbe D, Keliher D, Schilling B, Tracy A, et al (2018) Genomic correlates of response to immune checkpoint blockade in microsatellite-stable solid tumors. Nat Genet 50: 1271-1281. doi:10.1038/s41588-018-0200-2

O-charoenrat P, Rhys-Evans PH, Modjtahedi H, Eccles SA (2002) The role of c-erbB receptors and ligands in head and neck squamous cell carcinoma. Oral Oncol 38: 627-640. doi:10.1016/s1368-8375(02)00029-5

Pearlstein KA, Wang K, Amdur RJ, Shen CJ, Dagan R, Weiss J, Grilley-Olson JE, Zanation A, Hackman TG, Thorp BD, et al (2019) Quality of life for patients with favorable-risk HPV-associated oropharyngeal cancer after de-intensified chemoradiotherapy. Int I Radiat Oncol Biol Phys 103: 646-653. doi:10.1016/j.ijrobp.2018.10.033
Rooney MS, Shukla SA, Wu CJ, Getz G, Hacohen N (2015) Molecular and genetic properties of tumors associated with local immune cytolytic activity. Cell 160: 48-61. doi:10.1016/j.cell.2014.12.033

Samstein RM, Lee CH, Shoushtari AN, Hellmann MD, Shen R, Janjigian YY, Barron DA, Zehir A, Jordan EJ, Omuro A, et al (2019) Tumor mutational load predicts survival after immunotherapy across multiple cancer types. Nat Genet 51: 202-206. doi:10.1038/s41588-018-0312-8

Stransky N, Egloff AM, Tward AD, Kostic AD, Cibulskis K, Sivachenko A, Kryukov GV, Lawrence MS, Sougnez C, McKenna A, et al (2011) The mutational landscape of head and neck squamous cell carcinoma. Science 333: 1157-1160. doi:10.1126/science.1208130

Suit HD, Sedlacek RS, Silver G, Dosoretz D (1985) Pentobarbital anesthesia and the response of tumor and normal tissue in the $\mathrm{C} 3 \mathrm{Hf} /$ sed mouse to radiation. Radiat Res 104: 47-65. doi:10.2307/3576776

Takikita M, Xie R, Chung JY, Cho H, Ylaya K, Hong SM, Moskaluk CA, Hewitt SM (2011) Membranous expression of Her3 is associated with a decreased survival in head and neck squamous cell carcinoma. J Transl Med 9: 126. doi:10.1186/1479-5876-9-126

Van Allen EM, Lui VW, Egloff AM, Goetz EM, Li H, Johnson JT, Duvvuri U, Bauman JE, Stransky N, Zeng Y, et al (2015) Genomic correlate of exceptional erlotinib response in head and neck squamous cell carcinoma. JAMA Oncol 1: 238-244. doi:10.1001/jamaoncol.2015.34

Veatch JR, Lee SM, Fitzgibbon M, Chow IT, Jesernig B, Schmitt T, Kong YY, Kargl J, Houghton AM, Thompson JA, et al (2018) Tumor-infiltrating BRAFV600E-specific CD4+ T cells correlated with complete clinical response in melanoma. J Clin Invest 128: 1563-1568. doi:10.1172/ jci98689

Vigneswaran N, Williams MD (2014) Epidemiologic trends in head and neck cancer and aids in diagnosis. Oral Maxillofac Surg Clin North Am 26 123-141. doi:10.1016/j.coms.2014.01.001

Zehir A, Benayed R, Shah RH, Syed A, Middha S, Kim HR, Srinivasan P, Gao J, Chakravarty D, Devlin SM, et al (2017) Mutational landscape of metastatic cancer revealed from prospective clinical sequencing of 10,000 patients. Nat Med 23: 703-713. doi:10.1038/nm.4333

Zhang L, Castanaro C, Luan B, Yang K, Fan L, Fairhurst JL, Rafique A, Potocky TB, Shan J, Delfino FJ, et al (2014) ERBB3/HER2 signaling promotes resistance to EGFR blockade in head and neck and colorectal cancer models. Mol Cancer Ther 13: 1345-1355. doi:10.1158/1535-7163.mct-131033

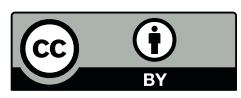

License: This article is available under a Creative Commons License (Attribution 4.0 International, as described at https://creativecommons.org/ licenses/by/4.0/). 